Aus dem Zentrum für Klinische Tiermedizin der Tierärztlichen Fakultät der Ludwig-Maximilians-Universität München

Arbeit angefertigt unter der Leitung von Univ.-Prof. Dr. med. vet. Johannes Hirschberger

\title{
Hämatologische und klinisch-chemische Referenzwerte für Hunde
}

Inaugural-Dissertation zur Erlangung der tiermedizinischen Doktorwürde der Tierärztlichen Fakultät

der Ludwig-Maximilians-Universität München

von

Anke Schäfers

aus Hamm

München 2013 
Gedruckt mit der Genehmigung der Tierärztlichen Fakultät der Ludwig-Maximilians-Universität München

Dekan: Univ.-Prof. Dr. Joachim Braun

Berichterstatter: Univ.-Prof. Dr. Johannes Hirschberger

Korreferent: Priv.-Doz. Dr. Monika Rinder

Tag der Promotion: 20. Juli 2013 
Meiner Familie (besonders meinem Vater) 


\section{INHALTSVERZEICHNIS}

INHALTSVERZEICHNIS IV

ABKÜRZUNGSVERZEICHNIS

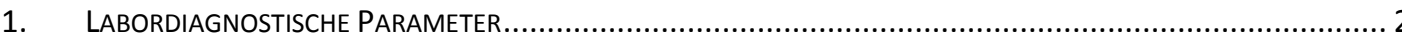

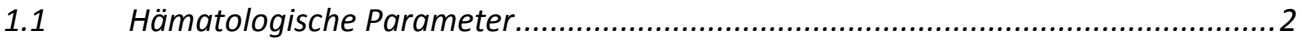

1.1.1 Leukozyten und Erythrozyten ................................................................ 2

1.1.2 Neutrophile, eosinophile und basophile Granulozyten ......................................

1.1.3 Lymphozyten, Monozyten und Thrombozyten ..............................................

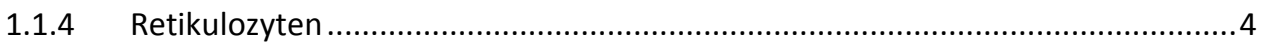

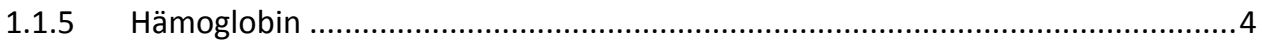

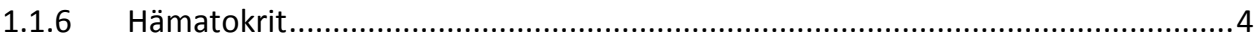

1.1.7 Mittleres Erythrozytenvolumen, Mittlere Hämoglobinkonzentration der

Erythrozyten und Mittlerer Hämoglobingehalt der Einzelerythrozyten ...........................5

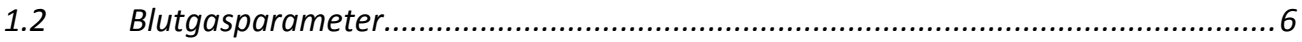

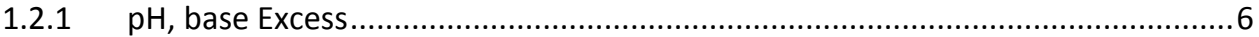

1.2.2 $\mathrm{pCO}_{2}, \mathrm{HCO}_{3}, \mathrm{TCO}_{2}$, Laktat und ionisiertes Kalzium ......................................6

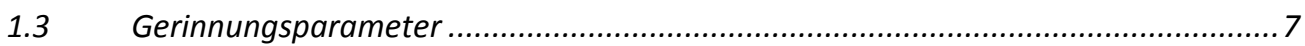

1.3.1 Optimierter Quick-Test............................................................................. 7

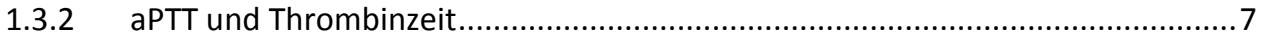

$1.4 \quad$ Klinisch-chemische Parameter....................................................................... 8

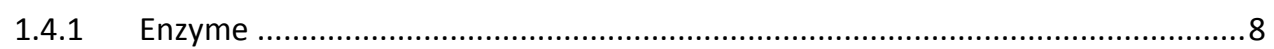

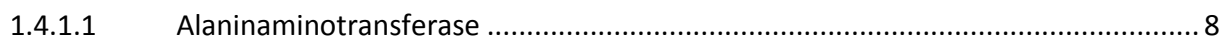

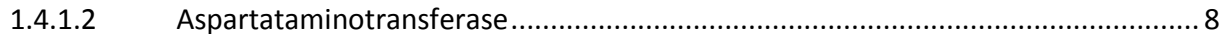

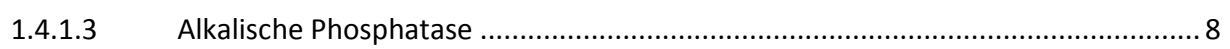

1.4.1.4 Glutamatdehydrogenase .............................................................................. 9

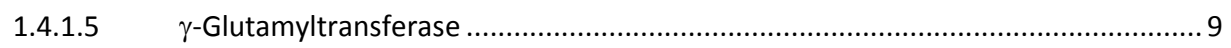

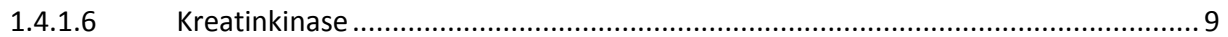

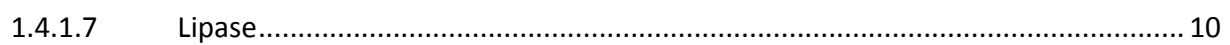

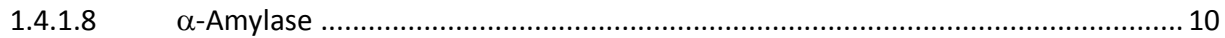

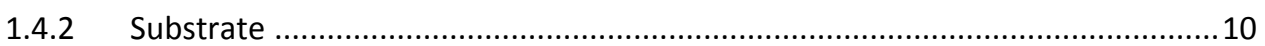

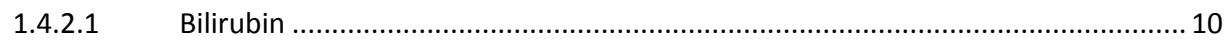

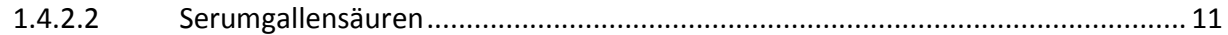

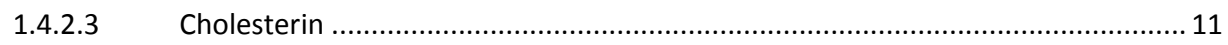

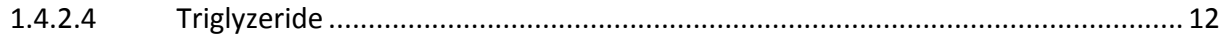

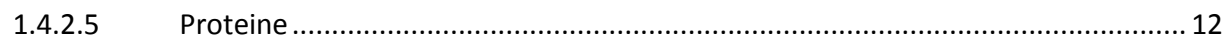

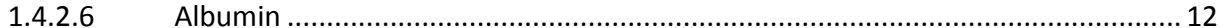




\begin{tabular}{|c|c|}
\hline 1.4.2.7 & 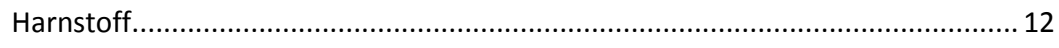 \\
\hline 1.4.2.8 & Kreatinin ................................... \\
\hline 1.4.2.9 & Glukose \\
\hline 1.4.2.10 & Fruktosamin \\
\hline El & (1) \\
\hline 1.4.3.1 & 14 \\
\hline 1.4.3.2 & Chlorid \\
\hline 1.4.3.3 & Natrium \\
\hline 1.4.3.4 & Kalium \\
\hline 1.4.3.5 & Kalzium \\
\hline
\end{tabular}

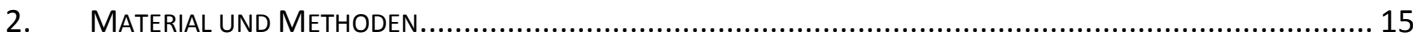

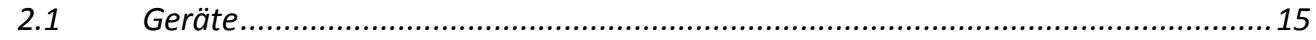

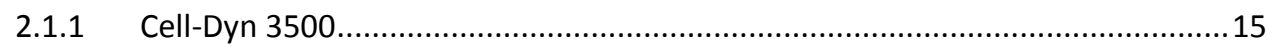

2.1.2 Hitachi 911 Automatic Analyzer ................................................................... 17

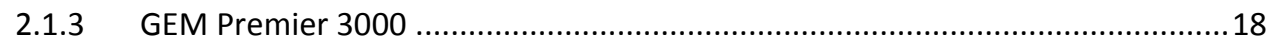

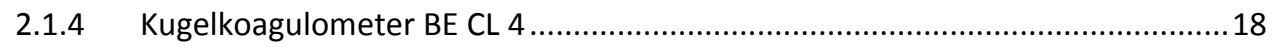

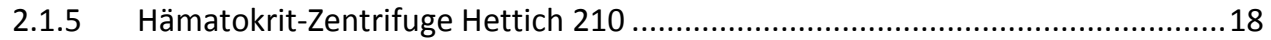

2.1.6 Färbebank Hema-Tek mit Modified Wright's strain .........................................19

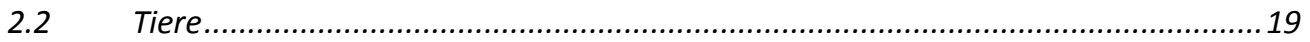

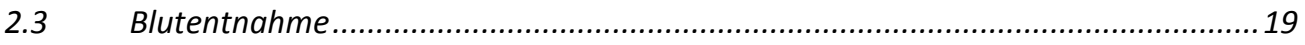

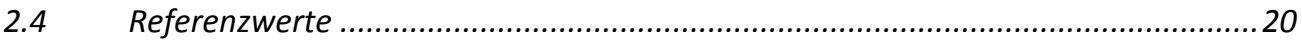

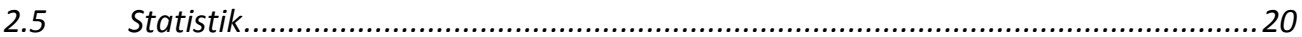

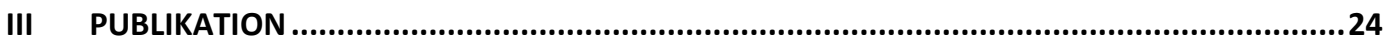

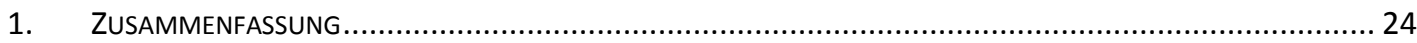

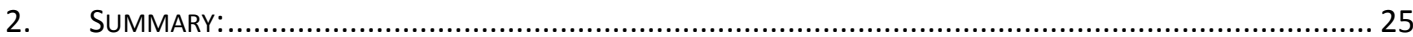

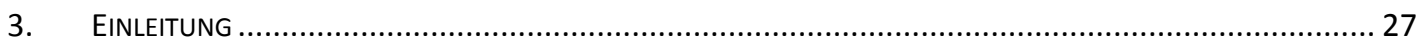

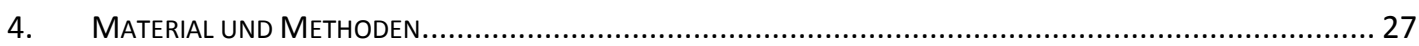

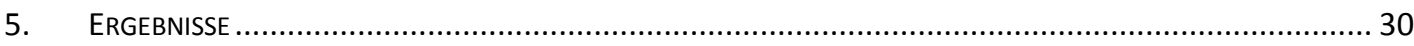

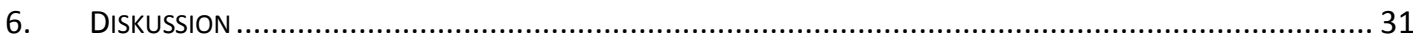

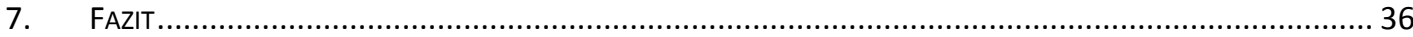

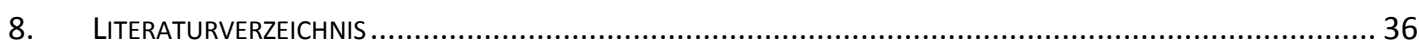

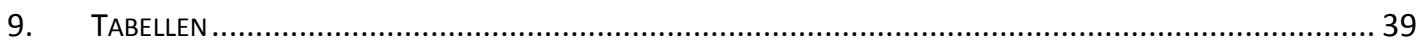

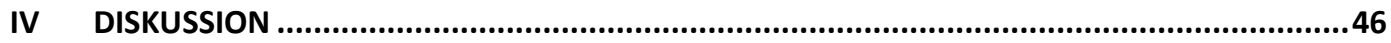

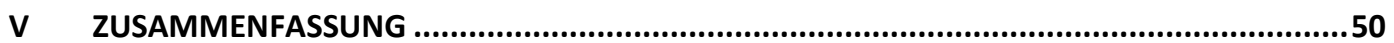

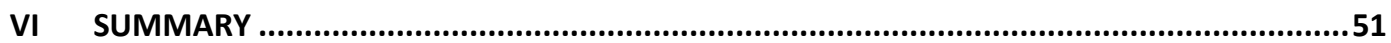

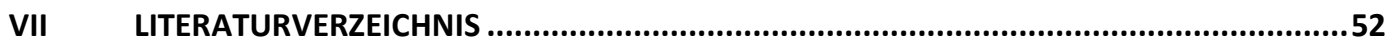

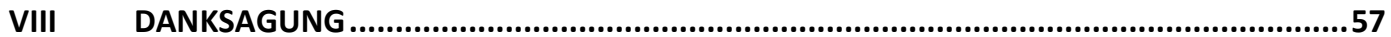




\section{ABKÜRZUNGSVERZEICHNIS}

\begin{tabular}{|c|c|}
\hline ALT & Alaninaminotransferase \\
\hline AP & Alkalische Phosphatase \\
\hline aPTT & Aktivierte partielle Thromboplastinzeit \\
\hline AST & Aspartataminotransferase \\
\hline ATP & Adenosintriphosphat \\
\hline $\mathrm{BE}$ & base Excess / Basenüberschuss \\
\hline bzw. & beziehungsweise \\
\hline ca. & circa \\
\hline $\mathrm{Ca}$ & Kalzium \\
\hline $\mathrm{CK}$ & Kreatinkinase \\
\hline $\mathrm{Cl}$ & Chlorid \\
\hline CSF & Cerebrospinal fluid Proben \\
\hline d & Tag \\
\hline$\gamma-\mathrm{GT}$ & Gamma-Glutamyltransferase \\
\hline fl & Femtoliter \\
\hline GLDH & Glutamatdehydrogenase \\
\hline $\mathrm{Hb}$ & Hämoglobin \\
\hline HGB & Hämoglobinbestimmung \\
\hline $\mathrm{HbE}$ & Färbekoeffizienten \\
\hline $\mathrm{HCO}_{3}$ & Hydrogencarbonat \\
\hline Hkt & Hämatokrit \\
\hline ISE & Ionen Selektive Elektrode \\
\hline
\end{tabular}


IU internationale Einheit (international Unit)

K Kalium

klin.-chem. klinisch-chemische Parameter

$\mathrm{kPa} \quad$ Kilopascal

m männlich

$\mathrm{MCH}$ mittlerer Hämoglobingehalt der Einzelerythrozyten

(mean corpuscular hemoglobin)

MCHC mittlere Hämoglobinkonzentration der Erythrozyten

(mean corpuscular hemoglobin concentration)

MCV mittleres Erythrozytenvolumen

(mean corpuscular volume)

MPV durchschnittliche Thrombozytenvolumen

mind. mindestens

mk männlich kastriert

n Anzahl der Werte/Tiere

$\mathrm{Na} \quad$ Natrium

P Phosphat

$\mathrm{pCO}_{2} \quad \mathrm{CO}_{2}$-Partialdruck

$\mathrm{pH} \quad$ Pondus Hydrogenii

PLT Thrombozyten

RBC Red blood cell

RDW Erythrozytenverteilungsbreite

segm. segmentkernige

SGS Serumgallensäure

SI Système international 
stab. Stabkernige

$\mathrm{TCO}_{2} \quad$ Gesamtkohlendioxid

U/min Umdrehungen pro Minute

V. Vena

w weiblich

WBC White blood cell

wk weiblich kastriert

z. B. zum Beispiel 


\section{I $\quad$ Einleitung und Zielsetzung}

In der Veterinärmedizin besonders in der Kleintierpraxis werden neben der klinischen Untersuchung auch die labordiagnostischen Möglichkeiten ausgeschöpft. Dadurch ist eine präzisere Diagnostik oder Bestätigung der klinischen Ergebnisse möglich. Die Referenzwerte beschreiben einen Rahmen indem der Messwert als ,normal“ bewertet wird.

Die immer weiter entwickelten Blutanalysegeräte analysieren heutzutage eine große Anzahl verschiedener Blutparameter und Differentialblutbilder. Die Analysegeräte, die in der Veterinärmedizin zum Einsatz kommen, sind meistens für den humanmedizinischen Gebrauch entwickelt worden. Durch die physiologische Abweichung von Tier- und Menschenblut ist es notwendig die Analysegeräte möglichst gut zu adaptieren.

Diese Arbeit wurde angefertigt um die bereits bestehenden Referenzwerte $\mathrm{zu}$ überprüfen oder gegebenenfalls zu korrigieren. Zusätzlich sind Abhängigkeiten der Referenzbereiche von Geschlecht, Alter und Futteraufnahme untersucht worden.

Die Ergebnisse ermöglichen es dem praktizierenden Tierarzt eine schnellere und genauere Diagnose zu stellen und durch die Einbeziehung der Abhängigkeiten der Faktoren ein Blutbild genauer interpretieren zu können.

Ziel war es eine umfangreiche Referenzwertanalyse der Hämatologie und klinischen Chemie von Hundeblutproben zu erarbeiten, sowie neue Erkenntnisse und Abhängigkeiten von verschiedenen Faktoren zu erhalten, die praxisrelevant eingesetzt werden können.

So ist es in Zukunft möglich Blutbilder besser dem Patienten und der klinischen Symptomatik zuzuordnen um eine genauere Diagnose zu stellen. 


\section{Literaturübersicht}

\section{Labordiagnostische Parameter}

Die labordiagnostischen Parameter werden unter hämatologischen Parametern und klinisch-chemischen Parametern zusammengefasst.

\subsection{Hämatologische Parameter}

Die hämatologischen Parameter wurden durch ein manuelles Differentialblutbild und den Cell-Dyn 3500 erstellt.

\subsubsection{Leukozyten und Erythrozyten}

Leukozyten (WBC) werden eingeteilt in Granulozyten, Lymphozyten, und Monozyten.

Im Blutplasma werden die weißen Blutzellen von der Bildungsstätte zum Wirkungsort transportiert. Nur diese Transportwege sind erfassbar und daher unterliegen die Zellzahlen großen Schwankungen (KRAFT et al., 2005).

Erythrozyten (RBC) entstehen bei Säugetieren aus kernhaltigen Erythroblasten und verlieren nach ihrer Reifung ihren Zellkern und die übrigen Zellorganellen. Es sind hochspezialisierte bikonkave Zellen, die tierartlich unterschiedliche Durchmesser von 3,9 bis 7,2 $\mu \mathrm{m}$ haben. Die Zellgröße steht im Verhältnis (bis auf wenige Ausnahmen) zur Körpergröße des Tieres. Der Gastransport (Atmungsfunktion des Blutes) mit Hilfe des roten Blutfarbstoffes Hämoglobin ist die Aufgabe der Erythrozyten (ENGELHARDT et al., 2000).

\subsubsection{Neutrophile, eosinophile und basophile Granulozyten}

Granulozyten zeichnen sich durch den Besitz charakteristischer Zytoplasmagranula und unterschiedliche Affinität gegenüber Farbstoffen aus. Sie werden im Knochenmark über mehrere Entwicklungsstufen ursprünglich aus den Myeloblasten gebildet.

Neutrophile Granulozyten bilden den größten Anteil mit bis zu $95 \%$. Chemotaktisch gelenkt gelangen sie durch amöboide Bewegung in der Regel als erste Abwehrzelle dem Ort der Gewebeschädigung um zunächst als Mikrophagen zu phagozytieren. 
Eosinophile Granulozyten sind artunterschiedlich mit bis $\mathrm{zu} 6 \%$ an der Leukozytenpopulation beteiligt. Sie sind länger im Blut als die Neutrophilen und gelangen über Diapedese ins Gewebe. Eosinophile Granulozyten sind amöboid beweglich und sind zur Phagozytose fähig. Durch die Freisetzung von Histaminase und Sulfatase wird Heparin und Histamin abgebaut, dies ermöglicht die Einleitung postentzündlicher Normalisierungsprozesse. Auf diese Weise werden die eosinophilen Granulozyten zu Mastzellantagonisten. Auf ihrer Oberfläche befinden sich Rezeptoren für $\operatorname{IgG}$, IgA, IgE, Komplementfaktoren, Zytokine, Chemokine und intrazelluläre Rezeptoren für Kortikoide. Im Gegensatz zur Immunantwort haben Eosinophile Granulozyten bei der Abwehr von Bakterien eine untergeordnete Funktion.

Basophile Granulozyten beteiligen sich mit bis $\mathrm{zu} \quad 0,5 \%$ an der Leukozytenpopulation. Ihre großen Zytoplasmagranula enthalten Histamin zur Vasodilatation und Heparin, das bei Gewebsschädigungen freigesetzt wird und so als eine Schutzwirkung gegen die disseminierte intravasale Gerinnung gesehen wird. Sie sind im Gegensatz zu den eosinophilen und den neutrophilen Granulozyten nicht amöboid beweglich. Sie setzten Botenstoffe für Eosinophile frei und sind dadurch indirekt an der Parasitenabwehr beteiligt (KRAFT et al., 2005; MICHL, 2005).

\subsubsection{Lymphozyten, Monozyten und Thrombozyten}

Lymphozyten werden aus Lymphoblasten gebildet. Sie befinden sich hauptsächlich im lymphatischen Gewebe. Ein kleiner Teil der Lymphozyten befindet sich auch im Blut und Knochenmark. Die differenzieren sich entweder zu T-Lymphozyten, die Träger der zellvermittelten Immunität sind oder BLymphozyten, die der Antikörperproduktion dienen. T- und B-Lymphozyten sind Träger der humoralen und spezifischen zellulären Abwehr. Die Lymphozyten werden auch als immunologisches Gedächtnis bezeichnet. Sie lassen sich nur mittels immunphänotypischer Untersuchungen unterscheiden (KRAFT et al., 2005; MICHL, 2005).

Monozyten entwickeln sich aus einer Vorläuferzelle der granulozytären Reihe. Sie entstehen im Knochenmark aus den Monoblasten über Promonozyten zum Monozyt. Sie sind $14-20 \mu \mathrm{m}$ große, vielgestaltige, kernhaltige Zellen, die 
phagozytieren. Sie sind für die unspezifische Abwehr zuständig. Monozyten zeichnen sich durch ihre amöboide Beweglichkeit und ihre ausgeprägte Migrationsfähigkeit aus. Differenziert werden wandernde Markophagen und sessile Makrophagen (Gewebsmakrophagen). In Stresssituationen kann die Monozytenzellzahl ansteigen. (KRAFT et al., 2005; MICHL, 2005)

Thrombozyten sind von Megakaryozyten im Knochenmark gebildete, kernlose, scheibenförmige Zellen, die endothelnah am Rand des Blutstromes zirkulieren. Es sind die wichtigsten Zellen für die Blutgerinnung. Bei einer Verletzung des Endothels haften sich die Thrombozyten an. Sie aggregieren und verkleben mit weiteren Thrombozyten und bilden einen Thrombus (KRAFT et al., 2005).

\subsubsection{Retikulozyten}

Retikulozyten entstehen im Knochenmark. Es sind Jugendformen der Erythrozyten. Mit zunehmender Reifung nimmt die charakteristische Substantia granulofilamentosa (netzartige, granuläre Zytoplasmastruktur) ab. Ihre Zellzahl und der Reifegrad im Blut geben einen Hinweis auf die erythropoetische Aktivität des Knochenmarks (WIESNER et al., 2000).

\subsubsection{Hämoglobin}

Das Hämoglobin ist der rote Blutfarbstoff in den Erythrozyten. Das Hämoglobinmolekül besteht aus vier Peptidketten, mit je einem Häm welches den Eisen-(II)-Protoporphyrin-Komplex (prosthetische Gruppe) darstellt. Globin bildet den Proteinanteil.

Die Peptidketten bestehend aus Alpha-, Beta-, Gamma- und Deltaketten haben die Fähigkeit Sauerstoff zu binden und zu transportieren. Der Abbau erfolgt über die Freisetzung des Hämoglobins aus den alternden oder geschädigten Erythrozyten.

Das freigesetzte Eisen wird wieder verwendet und der Rest in fettlösliches primäres Bilirubin umgebaut. In der Leber wird aus dem fettlöslichen, primären Bilirubin wasserlösliches, sekundäres Bilirubin, welches über die Galle ausgeschieden wird (KRAFT et al., 2005).

\subsubsection{Hämatokrit}

Er gibt den prozentualen Anteil der zellulären Bestandteile am gesamten 
Blutvolumen wieder. Der Hämatokritwert ist ein Relativwert und keine absolute Messgröße. Er gibt nur das Verhältnis Blutkörperchen zu Plasma an (KRAFT et al., 2005).

\subsubsection{Mittleres Erythrozytenvolumen, Mittlere Hämoglobinkonzentration der Erythrozyten und Mittlerer Hämoglobingehalt der Einzelerythrozyten}

Das mittlere Erythrozytenvolumen beschreibt das durchschnittliche Volumen der Erythrozyten und berechnet sich wie folgt:

$$
\mathrm{SI}=\operatorname{MCV}(\mathrm{fl})=\begin{gathered}
\text { Hämatokrit }(1 / \mathrm{l}) \times 10 \\
--------------- \\
\text { Erythrozytenzahl }(\mathrm{T} / \mathrm{l})
\end{gathered}
$$

MCH ist der mittlere Hämoglobingehalt des einzelnen Erythrozyten und gibt somit den durchschnittlichen Hämoglobingehalt des Einzelerythrozyten an. Man spricht auch vom Färbekoeffizienten (HbE). Die Genauigkeit hängt von einer korrekten Erythrozytenzählung ab. Die Unterscheidung von hypochromer, hyperchromer und normochromer Anämie ist mit dem MCH möglich.

Er errechnet sich wie folgt:

$$
\begin{aligned}
& \text { Hämoglobin (mmol/l) } \\
& \mathrm{MCH}(\mathrm{fmol})= \\
& \text { Erythrozytenzahl (T/l) }
\end{aligned}
$$

MCHC ist die mittlere Hämoglobinkonzentration der Erythrozyten. Mittels dieses Wertes lässt sich die hypochrome Anämie diagnostizieren.

Die Berechnungsformel ist wie folgt:

$$
\text { Hämoglobin (mmol/l) x } 100
$$

$\operatorname{MCHC}(\mathrm{mmol} / \mathrm{l})=$ 
(KRAFT et al., 2005).

\subsection{Blutgasparameter}

Die Blutgasparameter dienen der Diagnostik bei metabolischen und respiratorischen Alkalosen oder Azidosen (WIESNER et al., 2000).

\subsection{1 pH, base Excess}

Der pH-Wert (Säuregrad) ist der negativ dekadische Logarithmus der Wasserstoffionenkonzentration in mmol/l: $\mathrm{pH}=-\log \left[\mathrm{H}^{+}\right]$. Er ist eine Maßzahl für die Aktivität der freien Wasserstoffionen in einer Lösung (KRAFT et al., 2005; LEHNINGER et al. 1998).

Der Basenüberschuss (BE) des Vollblutes ist die titrierbare Azidität bei einem $\mathrm{CO}_{2}$-Partialdruck $\left(\mathrm{pCO}_{2}\right)$ von $40 \mathrm{mmHg}(=5,33 \mathrm{kPa})$ und einer Temperatur von $37^{\circ} \mathrm{C}$, wobei der Titrationsendpunkt einen $\mathrm{pH}$-Wert von 7,4 hat. Der BE bestimmt die Menge von Säure oder Base, die benötigt wird um einen Liter Blut auf einen physiologischen pH-Wert von 7,4 zu titrieren (KRAFT et al., 2005; WIESNER et al., 2000).

\subsection{2 $\mathrm{pCO}_{2}, \mathrm{HCO}_{3}, \mathrm{TCO}_{2}$, Laktat und ionisiertes Kalzium}

Im venösen und arteriellen Blut sind Gase in gelöster oder gebundener Form vorhanden. Diese Werte, wie der Kohlendioxidpartialdruck $\left(\mathrm{pCO}_{2}\right)$, Hydrogencarbonat $\left(\mathrm{HCO}_{3}\right)$, Gesamtkohlendioxid $\left(\mathrm{TCO}_{2}\right)$, werden gemessen und bilden zusammen mit dem pH-Wert eine Untersuchungsmöglichkeit des SäureBasen-Haushaltes zur Diagnostik der metabolischen und respiratorischen Alkalosen oder Azidosen (WILLARD et al., 2006).

Laktat ist die ionisierte Form der Milchsäure und ein Stoffwechselprodukt des postmortalen anaeroben Energiestoffwechsels bzw. ein wichtiges Produkt des Erythrozytenstoffwechsels. Ein Anstieg der Laktatkonzentration kann zu einer metabolischen Azidose führen (KRAFT et al., 2005; LEHNINGER et al., 1998).

Das ionisierte Kalzium ist $\mathrm{zu} 40 \%$ an Protein und zu $5 \%$ an anorganischen 
Säuren gebunden. Kalzium ist im Serum zu $55 \%$ ionisiert und dieser Anteil ist biologisch wirksam, darum ist die Messung des ionisierten Kalziums für eine differenzierte Beurteilung des Kalziumstoffwechsels wichtig. Die Konzentration hängt vom pH-Wert des Blutes ab, sie steigt an, bei sinkendem pH-Wert (KRAFT et al., 2005).

\subsection{Gerinnungsparameter}

Gerinnungsparameter sollen die Funktion der humoralen Komponenten der Koagulation bestimmen.

\subsubsection{Optimierter Quick-Test}

Der Quick-Test prüft die Faktoren VII, X, V, II und I (Fibrinogen). Ist die Plasmaaktivität eines dieser Faktoren unter $30 \%$ gesunken so verlängert sich die Gerinnungszeit. Um die Messung des Quick-Wertes beim Hund zu optimieren, wurde eine Empfindlichkeitssteigerung durch eine Verdünnung der Blutprobe und eine daraus resultierende Verlängerung der Gerinnungszeit erreicht. Das Testplasma wird vorverdünnt und extern zugesetztes Fibrinogen gewährleistet eine ausreichende Fibrinbildung und ermöglicht damit deren exakte messtechnische Erfassung (MISCHKE et al., 1997)

\subsection{2 aPTT und Thrombinzeit}

Aktivierte partielle Thromboplastinzeit (aPTT) untersucht die Aktivität der Faktoren XII, XI, IX, VIII, X, V, II und die Fibrinogenkonzentration. Der Zitratplasmaprobe wird ein Oberflächenaktivator (aPTT-Reagenz) bestehend aus zum Beispiel Kaolin, Celit oder Ellagsäure zugefügt, um so die Gerinnungskaskade auszulösen. Die Dauer von der Zugabe des Startreagenz bis zur Ausbildung eines Fibringerinnsels wird in Sekunden gemessen.

Die Thrombinzeit misst die Fibrinbildungszeit, die durch Zugabe eines Thrombinreagenz aus Fibrinogen gebildet wird. Es ist eine Untersuchung, die eine bessere Einordnung bei Verlängerung des Messergebnisses von aPTT liefert (ABBREDERIS, 2005). 


\subsection{Klinisch-chemische Parameter}

Die klinisch-chemischen Blutparameter werden unter Enzyme, Substrate und Elektrolyte zusammengefasst.

\subsubsection{Enzyme}

Enzyme haben wichtige Funktionen im Stoffwechsel. Es sind Biokatalysatoren, die das Tempo einer chemischen Reaktion im Organismus erhöhen aber nicht das Reaktionsgleichgewicht (KREUTZIG, 2001; LEHNINGER et al., 1998).

\subsubsection{Alaninaminotransferase}

Die Alaninaminotransferase (ALT) ist ein Enzym, das bei Hunden leberspezifisch ist. Sie kommt nur im Zytoplasma vor und ist nicht sehr sensitiv. Bereits bei Membrandesintegrationen erhöht sich ihre Aktivität (KRAFT et al., 2005).

\subsubsection{Aspartataminotransferase}

Die Aspartataminotransferase ist im Zytoplasma und in den Mitochondrien der Zellen, daher kommt es bei Membranschäden oder besonders bei Zellnekrosen zu einer Aktivitätserhöhung des Enzyms. Starke Aktivität der AST ist im Herzmuskel, Skelettmuskel und geringe Aktivität in der Leber festzustellen. Da die AST in verschiedenen Geweben und Organen vorkommt, ist sie nicht organspezifisch (KRAFT et al., 2005).

\subsubsection{Alkalische Phosphatase}

Die alkalischen Phosphatasen und die sauren Phosphatasen unterscheiden sich in ihrem pH-Optimum, bei dem sie reagieren. In fast allen Geweben ist die AP mit unterschiedlicher Aktivität nachzuweisen. Die AP spaltet organische Phosphorsäureester unter Freisetzung von Phosphat (WIESNER et al., 2000).

Sie ist an Membranstrukturen der Zelle gebunden. Beim Hund steigt die Aktivität besonders bei cholestatischen Zuständen an. Besonders bei Jungtieren ist eine hohe Enzymaktivität nachzuweisen, da das Enzym in den Osteoblasten enthalten ist. Dadurch ergibt sich eine altersabhängige Enzymaktivität, die bei der Beurteilung berücksichtigt werden muss (KRAFT et al., 2005).

Bei der erhitzten alkalischen Phoshatase wird ein Isoenzym, das durch 
Kortikosteroide aktiviert wird, nachgewiesen. Dieses Isoenzym wird durch die Erhitzung des Serums auf $65^{\circ} \mathrm{C}$ nicht inaktiviert. (TESKE et al. 1989)

\subsubsection{Glutamatdehydrogenase}

Die Glutamatdehydrogenase ist ein monolokuläres leberspezifisches Enzym, das an die Mitochondrienmatrix der Hepatozyten gebunden ist. Die höchste Aktivität befindet sich im zentritubulären Bereich des Leberläppchens. Die GLDH ist ein sehr sensitiver Parameter, bei geringgradigen, kurzfristigen Erhöhungen liegt nicht unbedingt ein Leberschaden vor. Erst bei starken Erhöhungen bis zum dreifachen des oberen Referenzbereiches kommen schwere Hepatopathien vor. Bei isolierten GLDH-Aktivitätserhöhungen ohne andere Leberenzymerhöhung sind Schäden im zentritubulären Bereich des Leberläppchens aufgetreten (KRAFT et al., 2005).

\subsubsection{5 $\gamma$-Glutamyltransferase}

Die $\gamma$-Glutamyltransferase gilt als leberspezifischer Messparameter und ist ein typischer Marker für Cholestasen, dabei reagiert sie aber träger als die alkalische Phosphatase und ALT. Dieses Enzym hat eine höhere Aktivität in der Niere als in der Leber. Die $\gamma$-Glutamyltransferase ist im Serum bei $-20^{\circ} \mathrm{C}$ bis zu einem Jahr stabil. Bei $4{ }^{\circ} \mathrm{C}$ ist sie bis zu drei Tage stabil (KRAFT et al., 2005; WILLARD et al., 2006).

\subsubsection{Kreatinkinase}

Dieses Enzym lässt sich in der Muskulatur, im Herzen und im Gehirn nachweisen. Labordiagnostisch ist die Kreatinkinaseaktivität im Muskel am bedeutsamsten. Beim Tier gilt sie als muskelspezifisches Enzym, da die Gehirn-Kreatinkinase nicht in das Blut übertritt.

Sie eignet sich zur Diagnostik von Muskelkrankheiten, Muskelschwäche und Herzmuskelkrankheiten, da die Kreatinkinase bei Zerstörung der Muskelzellen frei wird, da sie fast ausschließlich im Zytoplasma lokalisiert ist (KRAFT et al., 2005; HARTMANN, 1990) 


\subsubsection{Lipase}

Lipasen sind zusammen mit den Gallensäuren für die Fettverdauung zuständig. In der Labordiagnostik wird hauptsächlich die Pankreaslipase bestimmt. Zusätzlich kommt sie noch in der Magen- und Darmschleimhaut, in Fettgewebszellen, in der Gefäßintima und in der Leber vor. Zur Spaltung der Nahrungsfette wird Lipase in den Dünndarm sezerniert und dort durch die Salze der Gallensäuren aktiviert. Durch den emulgierenden Effekt auf Fette wird die Grenzfläche zwischen Öl und Wasser vergrößert und somit die Hydrolyse erleichtert. Die Synthese dieses zytoplasmatischen Enzyms findet im Pankreas und in den Azinuszellen an dem angelagerten Ribosomen des endoplasmatischen Retikulums statt.

Bei einer akuten Pankreatitis und Pankreasnekrose liegt ein deutlicher Konzentrationsanstieg vor. Sie ist wesentlich spezifischer als die Alpha-Amylase und steigt wesentlich später an. Sie sollte immer zusammen mit der AlphaAmylase gemessen werden (HARTMANN, 1990).

Liegt keine Pankreatitis vor, kann ein erhöhter Wert auch bei Niereninsuffizienz, Hepatopathien, diabetischer Ketoazidose, Dexamethason- oder Glucantimbehandlung vorkommen (KRAFT et al., 2005).

\subsubsection{8 $\alpha$-Amylase}

Die $\alpha$-Amylase wird im Pankreas, Speicheldrüse, Leber und im Dünndarm synthetisiert und sorgt im Dünndarm für die Kohlenhydratverdauung.

Die Sensitivität und Spezifität für die Diagnose einer Pankreatitis ist gering. Die Aktivität kann im Serum bei Raumtemperatur bis zu 7 Tage und bei $4^{\circ} \mathrm{C}$ bis zu einem Monat nachgewiesen werden. Die Aktivitätsstärke im Serum korreliert nicht mit dem Schweregrad einer Pankreatitis (KRAFT et al., 2005; WILARD et al., 2006)

\subsubsection{Substrate}

Substrate sind Verbindungen die bei biochemischen Reaktionen, wie zum Beispiel Biosynthesen oder Katalysen, mitwirken (LEHNINGER et al., 1998).

\subsubsection{Bilirubin}

Das Bilirubin (Gallenfarbstoff) ist ein Abbauprodukt von Hämoglobin, was erst 
als primäres, wasserunlösliches, lipidlösliches Bilirubin (Bilirubin I) an Albumin gebunden wird. Dieser Komplex wird an der Hepatozytenmembran durch Abspaltung des Albumins wieder getrennt und das Bilirubin I konjugiert mit Glucuronsäure zu wasserlöslichem Bilirubin-Diglucuronid (Bilirubin II), welches mit der Galle in den Dünndarm ausgeschieden wird. Das Gesamtbilirubin setzt sich aus dem Bilirubin I und dem Bilirubin II zusammen.

Geringgradige Konzentrationserhöhung im Blutserum beim Hund lassen sich nicht nachweisen, da die Nierenschwelle für Bilirubin II niedrig ist. Es wird zwischen prähepatischem oder hämolytischem Ikterus und posthepatischem oder cholestatischem Ikterus je nach Entstehungsort unterschieden (KRAFT et al., 2005).

\subsubsection{Serumgallensäuren}

Ein wichtiges Enzym für Digestion und Resorption von Lipiden aus dem Darm. Serumgallensäuren (SGS) werden in der Leber synthetisiert und gelangen mit der Galle in den Darm, wo ein geringer Teil mit dem Fäzes ausgeschieden wird. Der größte Teil wird proteingebunden wieder resorbiert und der Leber zugeführt.

Werden die Mikrovilli an der kanalikulären Seite des Hepatozyten geschädigt, können die Gallensäuren nicht mehr genügend ausgeschieden werden und akkumulieren im Blut. Dies hat zur Folge, dass die toxischen Eigenschaften zu Funktionsstörungen des Organismus führen.

Die Serumgallensäuren ist ein sehr sensitiver Parameter für Leber- und Gallengangserkrankungen (KRAFT et al., 2005).

\subsubsection{Cholesterin}

Cholesterin ist ein ungesättigter, einwertiger, hydroaromatischer Kohlenwasserstoff, der zu den Steroiden zählt. Die Hydroxylgruppe des Cholesterins kann in der Leber verestert werden oder frei sein. So entsteht das veresterte Cholesterin oder das sogenannte freie Cholesterin. Die Synthese findet in der Leber und verschiedenen anderen Geweben statt und ist in der Leber von der Nahrungsaufnahme abhängig. Die Ausscheidung des Cholesterins findet hauptsächlich über die Gallensäuren in den Darm statt (KRAFT et al., 2005; LEHNINGER et al., 1998). 


\subsubsection{Triglyzeride}

Triglyzeride oder Triacylglyzeride sind Ester des Glyzerins, eines dreiwertigen Alkohols, mit drei Fettsäuren und werden wegen Fehlens der elektrischen Ladung auch als Neutralfette bezeichnet. Stoffwechselkrankheiten können zu einer Entgleisung des Triglyzeridstoffwechsels führen (KRAFT et al., 2005).

\subsubsection{Proteine}

Die Plasmaproteine setzen sich aus über 100 verschiedenen zusammengefügten Proteinen zusammen, die im Gefäßsystem zirkulieren und teilweise auch im extravasalen Raum nachweisbar sind.

Bis auf die Immunglobuline werden hauptsächlich die Plasmaproteine in den Hepatozyten synthetisiert.

Bei einer Zunahme oder Abnahme des Proteinanteils im Serum spricht man von Hyperproteinämie bzw. Hypoproteinämie, die Ursache kann eine Dehydratation oder Hyperhydratation, dies ist aber keine echte Vermehrung bzw. Verminderung des Proteins. Des Weiteren gibt es zahlreiche relevante Ursachen für eine Hyperund Hypoproteinämie. Um absolute und relative Veränderungen zu erkennen hilft die Bestimmung des Hämatokrit (KRAFT et al., 2005).

\subsubsection{Albumin}

Albumine bilden die Hauptgruppe pflanzlicher und tierischer Proteine, die in Neutralsalzlösungen und Wasser löslich sind. Die Funktion eines Proteins wird durch seine dreidimensionale Struktur widergespiegelt. Albumin wird in der Leber synthetisiert und befindet sich in Blut und Lymphe. Seine Konzentration im Blutserum hängt vom Gleichgewicht zwischen Synthese, Abbau und Verlust ab (KRAFT et al., 2005, LEHNINGER et al., 1998).

\subsubsection{Harnstoff}

Harnstoff bildet tetragonale Prismen. Er ist in Alkohol und Wasser löslich und wirkt als schwache Base. Harnstoff wird aus Ammoniak in der Leber synthetisiert und ist das entgiftete Stoffwechselprodukt der Proteinverdauung. Über die Niere wird der Harnstoff hauptsächlich ausgeschieden aber auch in geringen Mengen über Schweiß und Verdauungssekrete (KRAFT et al., 2005; LEHNINGER et al., 
1998).

\subsubsection{Kreatinin}

Aus Kreatin und Phosphokreatin wird Kreatinin gebildet, es ist ein Produkt des endogenen Muskelstoffwechsels. Der Kreatininspiegel ist ein zuverlässiger Indikator für die renale Filtrationsrate, ein weiterer Vorteil ist, dass Kreatinin weder von dem Proteinmetabolismus noch von der Nahrungsaufnahme abhängig ist. Der Wert steht in Beziehung zur Muskelmasse des jeweiligen Tieres. In den Nieren wird Kreatinin glomerulär filtriert, ohne dass eine tubuläre Sekretion oder Rückresorption erfolgt, so dass eine Erhöhung des Kreatinins im Serumspiegel eine Einschränkung der Nierenfunktion bedeutet (ENGELHARDT et al., 2000; KRAFT et al., 2005).

\subsubsection{Glukose}

Glukose kommt in freier und gebundener Form in pflanzlichen und tierischen Geweben vor. Glukose ist ein Monosaccharid und am Aufbau von Maltose, Saccharose, Lactose, Stärke und anderen Kohlenhydraten beteiligt. Sie wird in der Leber durch die Glukoneogenese und Glykogenolyse gebildet und über das Futter aufgenommen, wodurch ein Einfluss der letzten Futteraufnahme auf die Glukosekonzentration im Serum besteht. Sie ist in vielen tierischen Zellen eine leicht verwertbare Hexose, die in einer bestimmten Konzentration im Blutplasma als sogenannter Blutzucker vorkommt (ENGELHARDT et al., 2000; LEHNINGER et al., 1998).

\subsubsection{Fruktosamin}

Die Fruktosaminkonzentration ist ein guter Indikator für die Diabetisdiagnostik. Eine länger bestehende Hyperglykämie, lässt den Frukosaminwert ansteigen. So kann ein Diabetis mellitus von einer passageren Hyperglykämie unterschieden werden. Auch kann mit Hilfe der Fruktosaminkonzentration die Einstellung mit Insulin eines diabetischen Patienten erleichtert werden (KRAFT et al., 2005).

\subsubsection{Elektrolyte}

Elektrolyte sind Verbindungen aus Säuren, Basen oder Salze, die in wässrigen Lösungen mehr oder weniger stark in freibeweglichen Ionen dissoziieren und der 
Elektrolyse unterzogen werden können (WIESNER et al., 2000).

\subsubsection{Phosphor}

Phosphor kommt im Blut als anorganisches Phosphat, Phospholipid und als organischer Ester vor. Es kommt zu 75 - 85\% im Knochen und 15 - 25\% in den Wiechteilgeweben vor. Das Serum-Phosphat ist diagnostisch wichtig. Die löslichen Phosphate bilden Puffersubstanzen, die zur Herstellung von Puffern dienen und auch in Puffersystemen des Organismus enthalten sind (ENGELHARDT et al., 2000; KRAFT et al., 2005).

\subsubsection{Chlorid}

Chlorid ist zusammen mit Natrium für den osmotischen Druck im Extrazellularraum verantwortlich. Etwa $85 \%$ liegen beim Tier im Extrazellularraum vor. Chloride sind zum größten Teil gut wasserlöslich und sind teilweise auch für die Erregbarkeit der Sinnes- und Nervenzellen mit verantwortlich (ENGELHARDT et al., 2000; KRAFT et al., 2005).

\subsubsection{Natrium}

Natrium liegt in der höchsten Konzentration im Extrazellularraum vor und ist für den osmotischen Druck verantwortlich. Die Zu- oder Abnahme der Osmolarität erfolgt durch eine Änderung der Natriumkonzentration(im Extrazellularraum). Natrium wird zum größten Teil über die Nieren, der Rest über die Schweißdrüsen und den Kot ausgeschieden. Änderungen des Natriumhaushaltes können hypertone, isotone oder hypotone Hyperhydratation bzw./oder Dehydratation zur Folge haben (KRAFT et al., 2005).

\subsubsection{Kalium}

Es befinden sich bis zu 98\% des Gesamtkörperkaliums um Intrazellularraum. Kalium kann sich mit Hilfe der Natrium-Kalium-ATPase zwischen dem Extraund Intrazellularraum bewegen und bestimmt somit auch den osmotischen Druck. Längerfristige Elektrolytverluste haben zur Folge, dass Kalium für den Extrazellularraum aus der Muskulatur ersetzt wird. Drei Kaliumionen werden aus der Zelle gegen zwei Natriumionen und ein Wasserstoffion getauscht, dies führt zu einer Azidose der Zelle, während sich eine hypokaliämische Alkalose durch die erhöhte renale Chloridausscheidung aufgrund der Erhöhung der 
extrazellularen Bicarbonatkonzentration bildet. Bei einer Hypokaliämie ist die Kaliumkonzentrationsveränderung extrazellulär größer als intrazellulär. Der Quotient steigt und somit auch das Ruhepotential. Der Quotient intra- zu extrazellulärem Kalium nimmt bei einer Hyperkaliämie ab (KRAFT et al., 2005).

\subsubsection{Kalzium}

Kalzium zusammen mit anderen Mineralstoffen verleiht dem Knochen Härte und Formbeständigkeit. Es beeinflusst die Erregbarkeit von Muskeln und Nerven, sowie den Stoffwechsel, die Blutgerinnung und die Durchlässigkeit von biologischen Membranen. Die Kontraktion des Herzen wird durch Kalzium ausgelöst. Eine wichtige Voraussetzung für ein gesundes Herz ist die langfristige Feinreglung der zellulären Calciumhomöostase. Kalzium-Ionen sind wichtige Mediatoren zwischen Erregung und Kontraktion der Muskulatur durch die Wechselwirkung zwischen Myosin und Actin und der damit verbundenen aktivierten ATPase (ENGELHARDT et al., 2000).

\section{Material und Methoden}

\subsection{Geräte}

Messgeräte dienen der Bearbeitung und Analyse von Blutproben in medizinischen Laboren.

\subsubsection{Cell-Dyn 3500}

In Deutschland wird der Cell-Dyn 3500 von der Firma Abbott GmbH Diagnostika (Wiesbaden) vertrieben und ist seit 1993 auf dem Markt. Dieses Gerät ist ein vollautomatisches, elektronisches Blutanalysesystem, welches die Impedanzmessmethode mit der Durchflusszytometrie vereint. Der Cell-Dyn 3500 setzt sich aus vier Baugruppen zusammen: der Analyseeinheit, der Datenstation, einem Grafikdrucker und vier externen Reagenzienbehälter.

Zur Analyse der hämatologischen Parameter werden im Gerät vier unabhängige Messungen vorgenommen.

- Im optischen Kanal werden die Daten für die optische Leukozytenzählung und für die Leukozytendifferenzierung gemessen. 
- In dem ersten Widerstandsmesskanal erfolgt die Leukozytenwiderstandsmessung.

- In dem zweiten Widerstandsmesskanal werden die Thrombozyten (PLT) und die Erythrozyten (RBC) gemessen.

- In dem Spektrophotometriekanal wird die Hämoglobinkonzentration ermittelt.

Die Impedanzmessung ist für die Zellzählung und Größenbestimmung der Erythrozyten und Thrombozyten. Die Zählung und Differenzierung der Leukozyten ist eine Kombination der optischen Messung mit der Impedanzmessung. Die Hämoglobinbestimmung (HGB) erfolgt photometrisch. Mit den gängigen Formeln errechnet der Cell-Dyn 3500 den Hämatokrit (HKT), das mittlere korpuskuläre Hämoglobin $(\mathrm{MCH})$ und die mittlere korpuskuläre Hämoglobinkonzentration (MCHC). Aus den RBC- bzw. PLT-Histogrammen werden das mittlere Erythrozyteneinzelvolumen (MCV), die Erythrozytenverteilungsbreite (RDW) und das durchschnittliche Thrombozytenvolumen (MPV) abgeleitet.

Das Gerät benötigt pro Messzyklus $130 \mu 1 \mathrm{~K}_{3}$ EDTA-Blut oder Vollblut, welches eingesaugt wird. Der Cell-Dyn 3500 arbeitet mit drei Probenverdünnungen.

- WBC optical count (WOC)-Verdünnung

- $\quad$ WBC impedance count (WIC)- / HGB-Verdünnung

- $\quad$ RBC- / PLT-Verdünnung

Die benötigten Reagenzien sind:

- Sheath-Reagenz (REF 99311-01) verdünnt die Leukozyten

- Diluent (REF 99231-01) Verdünnungslösung der Leukozyten, der RBC, der PLT und der HGB-Messung

- WIC / HGB lyse (REF 99431-01) für die Lyse der RBC

- Detergent (REF 99321-01) klare Lösung für 0-Wert Kalibrierung, Spülung der Zählkammer sowie zur Minimierung der Luftblasen

- Enzymatic Cleaner (REF 93641-01) zur Entfernung von Proteinrückständen und Reinigung des Gerätes

Der Cell-Dyn 3500 ist mit einer Veterinärsoftware erhältlich, die aber keine vorgegebenen Grenzwerte für den Messbereich hat, sondern variable Schwellenwerte. Ein Barcode-Reader und einen Probenspeicher für 9999 Proben sind vorhanden. Markierungen für atypische Verteilungen, atypische Parameter, 
atypische Zellpopulationen und interpretierende Hinweise ermöglicht die Software. Die Kalibration führt der Hersteller bei Installation durch, weitere Kalibrationen werden bei der Wartung und beim Austausch von Geräteteilen durch den Techniker durchgeführt. Der Cell-Dyn 3500 führt während des Startvorganges eine Leerwertmessung durch und prüft alle wichtigen Funktionen und meldet gefundene Fehler. Die Ergebnisse werden auf einem Bildschirm dargestellt und können auch ausgedruckt werden (ABBOTT, 2000).

\subsubsection{Hitachi 911 Automatic Analyzer}

Der Hitachi 911 (Firma Roche, Mannheim, Germany) ist ein vollautomatisches Analysegerät. Dieses Gerät besteht aus folgenden Einheiten:

- Analytische Einheit mit einem ISE System (Ionen Selektive Elektrode), einem Prozessor (CPU) und einer photometrischen Messzelle

- Kontrolleinheit mit Monitor, Keyboard und einem Drucker für die Ausgabe der Messergebnisse

Dieses Gerät kann verschiedene Proben analysieren. Verwendet werden können Urin, Serum, Plasma und Cerebrospinal fluid Proben (CSF).

Das Probensystem besteht aus dem Probenteller mit Pipetierarm und Waschstation. Der Probenteller hat drei Ringe mit jeweils 50, 40 und 25 Plätzen, die für verschiedene Lösungen, Kalibratoren, Kontrollen und Proben sind.

Der Pepetierarm transferiert selbstständig die Proben vom Teller in die Reaktionskammer. Das Reagenziensystem besteht aus zwei Tellern mit Deckel und insgesamt 66 Plätzen. Die 120 Plastikküvetten der Reaktionsteller sind in einem auf $37^{\circ} \mathrm{C}$ konstant temperierten Reaktionsbad mit deionisiertem Wasser, welches beim Verdampfen durch die angeschlossene Wasserversorgung wieder aufgefüllt wird.

Der Hitachi 911 hat ein photometrisches Messsystem, welches alle 20 Sekunden einen Extinktionswert liefern kann. Die Photometerlampe befindet sich in einem gleichbleibend temperierten Wasserbad, um so einen gleichmäßigen Energieausstoß zu gewährleisten.

Die Ionen Selektive Elektrode ermöglicht es, Natrium, Kalium und Chlorid haltige Proben zu messen. Vor jeder Messung wird mit einer Referenzlösung eine ein-Punkt Kalibrierung durchgeführt. Das Gerät benötigt $450 \mu$ l einer Probe und 
verdünnt diese im Verhältnis 1:31 in der Verdünnungskammer. In der Messkammer werden dann die Potentiale gemessen. Anschließend wird die Messzelle durch die interne Referenzlösung gewaschen. Je nach Anforderung werden die Analysedaten einzeln oder hintereinander vom Hitachi 911 gesendet und dann über die Kontrolleinheit zum Drucken gegeben (HOLCAK \& RIEHS, 2003; ZAMAN et al. 1993).

\subsubsection{GEM Premier 3000}

Dieses medizinische, vollautomatische Analysegerät von Instrumentation Laboratory $\mathrm{GmbH}$ Kirchheim bei München benötigt ca. $50 \mathrm{U} / \mathrm{ml}$ lithiumheparinisiertes Blut oder Vollblut, um Blutgas, Elektrolyte und den Sauerstoffgehalt zu messen. Sämtliche Sensoren, Schläuche und Reagenzien befinden sich nur in einer Kassette. Die direkte Potentiometrie analysiert den $\mathrm{pH}$ Wert, $\mathrm{pCO}_{2}$, Natrium Kalium und ionisiertes Calcium. Über die Aperometrie wird $\mathrm{pO}_{2}$, Glucose und Laktat ermittelt. Der Hämatokrit wird mit Hilfe der Konduktometrie bestimmt. Das Ergebnis wird nach spätestens 3 Minuten ausgedruckt (NOTFALLLABOR, 2008).

\subsubsection{Kugelkoagulometer BE CL 4}

Das CL 4 (Behnk Elektronik GmbH \& Co, Norderstedt, Germany) hat vier Messkanäle. Der motorbetriebene Magnet jedes Messkanals bringt die Magnetkugel unterhalb jedes Kanals zum Rotieren. Die Bewegung der Kugel kommt durch Fibrinfäden zum Stillstand, die sich beim Einsetzen der Gerinnung bilden. Ein Sensor registriert den Stillstand der Kugel und die Zeitmessung wird gestoppt.

\subsubsection{Hämatokrit-Zentrifuge Hettich 210}

Der Hämatokrit wurde manuell nach Angaben des Herstellers mit der Tischzentrifuge von Hettich bestimmt. Pro Lauf können bis $\mathrm{zu} 24$ Hämatokritkapillaren auf den Drehteller eingelegt werden. Diese werden mit Blut gefüllt, anschließend einseitig mit Kitt verschlossen und eingelegt. Die Drehzahl des Tellers beträgt 13000 Umdrehungen pro Minute. Auf einer Auswertskala wird nach der Zentrifugation der Kapillaren der Hämatokrit abgelesen (HETTICH et al., 2008). 


\subsubsection{Färbebank Hema-Tek mit Modified Wright's strain}

Die Objektträger wurden nach Angaben des Herstellers mit dem Blutausstrichfärbeautomat von Hema-Tek (Bayer, Leverkusen, Germany) vorgenommen. Es ist ein vollautomatisches Gerät speziell zum Färben von Blutausstrichen. Pro Stunde können bis zu 60 Färbungen gemacht werden. Ein kontinuierliches Nachlegen der Objektträger während des Betriebes ist möglich. Das Fassungsvermögen beträgt bis zu 25 Objektträger. Für die gleichbleibende Farbqualität sorgt ein sparsamer Kapillarraum, der immer frische Reagenzien nachfüllt. Die getrockneten Ausstriche fallen in einen Auffangbehälter (VOGEL Medizinische Technik und Elektronik, 2009).

\section{$2.2 \quad$ Tiere}

In dieser Studie wurden die Blutbilder von Hunden ausgewertet. Durch Domestikation entwickelte sich der Haushund (Canis lupus familiaris) nach wissenschaftlichen Schätzungen vor ca. 10.000 Jahren aus dem Wolf (Canis lupus) (AHNE et al., 2000). In unserer heutigen Gesellschaft sind Haushunde nach Katzen die beliebtesten Haustiere. Laut Schätzungen gibt es weltweit circa 500 Millionen Haushunde, wovon über 5 Millionen in Deutschland leben. Diese domestizierten Hunde sind an das Zusammenleben mit dem Menschen und die menschliche Gesellschaft gewöhnt und gehen mit ihnen eine soziale Beziehung ein. Der Hund ist rassetypisch für verschiedene Aufgaben prädisponiert. Sie bewachen, jagen, hüten, suchen, begleiten und retten Menschenleben. In den meisten Fällen sind Hunde ein geliebtes und geschätztes Familienmitglied. (FENNELL, 2008)

\subsection{Blutentnahme}

Venöses Blut aus der Vena cephalica des distalen Unterarms bei Hund und Katze wird für die meisten Untersuchungen verwendet. Weitere Entnahmestellen beim Hund sind die V. saphena lateralis und die V. jugularis. Die Punktion der Vene sowie das Stauen der Vene sollte so schonend wie möglich geschehen, damit keine Gewebsschäden ausgelöst werden. Das Blut soll frei fließend an der Gefäßwand entlang aufgefangen werden (KRAFT et al., 2005). 


\section{$2.4 \quad$ Referenzwerte}

Referenzwerte umfassen einen Parameterbereich, der als normal interpretiert wird (WIESNER, 2000). Ermittelt werden sie durch Untersuchungen an gesunden Tieren. Referenzbereiche sind erforderlich, um Laborbefunde $\mathrm{zu}$ beurteilen. Verschiedene Geräte oder Untersuchungsmethoden weisen unterschiedliche Referenzbereiche auf, darum ist es wichtig, dass jedes Labor eigene Referenzwerte ermittelt (KRAFT et al., 2005).

Die Erstellung eines Referenzbereiches erfolgt unter exakt definierten Bedingungen von einer ausreichend beschriebenen Gruppe von Probanden. Aus den gesammelten Daten wird mit einer bestimmten mathematisch-statistischen Methode das Referenzintervall ermittelt (KRAFT et al., 2005). Das Über- oder Unterschreiten der Grenzwerte deutet in der Regel auf einen pathologischen Befund hin (DE GRUYTER et al., 2002).

\section{$2.5 \quad$ Statistik}

Die Statistik ist ein Hilfsmittel zur Informationsgewinnung und eine Basis der Entscheidungsfindung. Der Ablauf einer wissenschaftlichen Arbeit unter Einbezug einer statistischen Untersuchung sollte klar strukturiert sein (LAUFNER, 2000).

\begin{tabular}{|l|rl|}
\hline 1. Planung & $\bullet$ & Zielformulierung \\
& $\bullet$ & Klarstrukturierter Plan der \\
& & Durchführungsmethoden \\
& & \\
\hline 2. Datenerhebung & $\bullet$ & Sammlung von Daten \\
\hline 3. Datenauswertung & $\bullet$ & Beschreibende Statistik \\
& $\bullet$ & Häufigkeitsverteilung \\
& $\bullet$ & Lage- und Streuparameter \\
& $\bullet$ & Erstellung von Tabellen und \\
& & graphische Darstellungen \\
\hline 4. Datenanalyse & $\bullet$ & Schließende Statistik \\
& $\bullet$ & Analysieren von \\
\hline
\end{tabular}




\begin{tabular}{|c|c|}
\hline & $\begin{array}{ll} & \text { Fragenkomplexen } \\
\text { - } & \text { Abhängigkeitsanalysen } \\
\text { - } & \text { Multivariate Analysen }\end{array}$ \\
\hline 5. Interpretation der Ergebnisse & $\begin{array}{l}\text { - Die Ausgangs-/ } \\
\text { Zielformulierung soll anhand } \\
\text { der Ergebnisse erklärt werden } \\
\text { können }\end{array}$ \\
\hline
\end{tabular}

Tab.1: Ablaufsplan einer statistischen Untersuchung

Das Ziel der vorliegenden Arbeit bezieht sich auf die Referenzwertbestimmung von Blutparametern und klinisch-chemischen Parametern unter Berücksichtung von möglichen Abhängigkeiten verschiedener Faktoren.

Nach der Datenauswertung, die zur ersten Darstellung der vielen Datenreihen, mit Hilfe von Lage- und Streuparametern dient, können die Analyseinstrumente zur Abhängigkeitsanalyse gewählt werden. Die Entscheidung, welche Analysevarianten ausgesucht werden, hängt von der Zielformulierung, des Skalenniveaus und der Menge der Faktoren ab (BACKHAUS et al., 1996). Viele Analysen verlangen bestimmte Voraussetzungen zur Durchführung, sind diese nicht gegeben verfälschen diese die Ergebnisse. Daher sollte die Auswahl gut überlegt sein, damit ein repräsentatives Ergebnis folgt (EIMER, 1978).

Ein erster Schritt kann die Ausreißereliminierung nach Henry und Reed sein. Es stellt keine Vorraussetzung dar, kann aber einzelne Werte in der Datenreihe, die sich von anderen Werten unterscheiden und die statistische Maße gegebenenfalls beeinflussen und somit die Ergebnisse verfälschen, eliminieren (HENRY et al., 1974).

Ein häufig gewähltes Verfahren ist die Varianzanalyse. Sie untersucht die 
Abhängigkeit einer Variablen von anderen gegebenen Variablen (BACKHAUS et al., 1996). Die Varianzanalyse kann nur unter bestimmten Vorraussetzungen durchgeführt werden. Dazu zählen die Normalverteilung, die Varianzhomogenität und die Zufallsauswahl (MEFFERT, 1992). Wird eine Vorraussetzung nicht erfüllt, muss eine alternative Analyse in Betracht gezogen werden.

Mit Hilfe verschiedener Testverfahren kann die Normalverteilung überprüft werden. Hierzu dienen z.B. der Kolmogoroff-Smirnow-Test, Lillefors-Test, Kurtosius/Schiefe, Chiquadrat-Test und der Nullklassentest (SCHACH \& SCHÄFER, 1978). Da die vorliegenden Daten nicht normalverteilt sind, kann die Varianzanalyse nicht angewandt werden.

Es muss ein nicht-parametrisches Testverfahren gewählt werden. Diese verlangen keine Normalverteilung und sind robuster gegenüber Ausreißern. Es wird nicht mit den Messwerten gearbeitet, sondern mit den Rangwerten (LAUFNER, 2000).

Die hier gewählten Testverfahren zur Abhängigkeitsüberprüfung sind der MannWhitney-U-Test und der Kruskall-Wallis Test. Bei beiden Testverfahren wurde $\alpha=0,05 / 100$ eingesetzt (Bonferoni-Korrektur). Bei dem U-Test, nach Mann und Whitney, werden zwei Stichproben untereinander verglichen. Bei dem KruskalWallis-Test hingegen, können mehr als zwei unabhängige Stichproben miteinander verglichen werden (GUGGENMOOS-HOLZMANN \& WERNECKE, 1995).

Die Referenzbereiche werden hier mit Hilfe der Perzentilentechnik bestimmt, in der Regel werden 95 Prozent der gesunden Masse als normal angesehen. Das heißt, es wird bei einem zweiseitigen Referenzbereich das 2,5\% Perzentil und das 97,5\% Perzentil bestimmt. Der zweiseitige Referenzbereich eliminiert je von den oberen und von den unteren Werten 2,5\%. Die Auswahl dieser Einschränkung muss biologisch sinnvoll sein. Diese Methode hat sich in der Medizin bewährt und wird meistens angewandt (HARTMANN, 1990; KRAFT et al., 2005; WILLARD et al. 2004). Weitere mögliche Bereichsbestimmungen sind Ranges und die klassische Bereichsbestimmung. Die Ranges geben lediglich die Spannweite der Datenreihen wieder, somit werden alle gesunden Tiere als normal angesehen. Die Ausnahme, dass auch ein gesundes Tier einen nicht normalen 
Wert aufweisen könnte, wird nicht berücksichtigt. Die klassische Bereichsbestimmung setzt eine Normalverteilung voraus, die im vorliegenden Fall nicht gegeben ist (HENRY et al., 1974). 


\title{
III Publikation
}

Hämatologische und klinisch-chemische Referenzwerte für Hunde

\author{
A. Schäfers ${ }^{1,4}$, S. Meierhans ${ }^{2}$, C. Sauter-Louis ${ }^{3}$, K. Hartmann ${ }^{1}$, J. \\ Hirschberger ${ }^{1}$ \\ ${ }^{1}$ Medizinische Kleintierklinik, Ludwig-Maximilians-Universität; ${ }^{2}$ Werne; ${ }^{3}$ Klinik \\ für Wiederkäuer, Ludwig-Maximilians-Universität München \\ ${ }^{4}$ Korrespondenzadresse \\ Anke Schäfers \\ Herzog-Egbert-Str. 1, 59510 Lippetal \\ Email: info@tierarztpraxis-herzfeld.de
}

Schlüsselwörter

Referenzwerte, Hunde, Hämatologie, klinische Chemie, Blutgas, Koagulometrie, Cell-Dyn 3500, Hitachi 911, GEM Premier 3000

\section{Zusammenfassung}

Ziel dieser Studie war, Referenzwerte für hämatologische und blutchemische Parameter bei adulten Hunden $\mathrm{zu}$ etablieren und diese hinsichtlich einer Abhängigkeit von Alter, Geschlecht und Fütterung zu überprüfen. Material und Methoden: Bei den Probanden handelte es sich um 508 klinisch gesunde Hunde beiderlei Geschlechts im Alter von $\leq 1$ bis 17 Jahren, die unterschiedlichen Rassen angehörten. Für die Bestimmung der Referenzbereiche wurden die Werte von 396 Hunden mit einem Alter von 1-9 Jahren herangezogen. Zur hämatologischen und blutchemischen Untersuchung der Blutproben dienten folgende Geräte: Cell-Dyn 3500, Kugelkoagulometer BE CL 4, Blutgasanalysegerät GEM Premier 3000, Elecsys 1010 und der Hitachi 911. Die Referenzwerte wurden statistisch mit SPSS 14 erstellt. Ergebnisse: Bei 75\% der Parameter unterschieden sich die Resultate unwesentlich von bestehenden Referenzbereichen. Abweichungen ergaben sich für folgende Parameter: eosinophile und basophile Granulozyten, Monozyten, Alaninaminotransferase (ALT), alkalische Phosphatase (AP), Glutamatdehydrogenase (GLDH), Lipase, Kreatinkinase, Bilirubin sowie Kreatinin. Der Referenzbereich der eosinophilen Granulozyten, Monozyten sowie der GLDH lag höher, als in der Literatur angegeben. Ein niedrigerer Referenzbereich im Vergleich zur Literaturangaben 
war für die basophilen Granulozyten festzustellen. Bei den Enzymen ALT, AP und Lipase differierten die Referenzbereiche der jungen $(<1 \mathrm{Jahr})$ und alten Hunde ( $\geq 10$ Jahre) signifikant von den Referenzbereichen der 1-9 Jahre alten Tiere. Schlussfolgerung und klinische Relevanz: Referenzwerte sollten in regelmäßigen Zeitintervallen überprüft werden, da sich durch fortschreitende Entwicklung und neue Erkenntnisse einige Faktoren der Bestimmung, vor allem Geräte und Methoden, ändern.

\section{Key words}

Laboratory diagnostics, haematology, serum chemistry, blood gas analysis, coagulometry, Cell-Dyn 3500, Hitachi 911, GEM Premier 3000

\section{Summary:}

Objective: The aim of the study was to establish reference values for haematological and clinical-chemical parameters in adult dogs and to evaluate if they are influenced by age, sex and feeding. Material and methods: The study population consisted of 508 clinically healthy dogs of both genders and different breeds in the age between $\leq 1$ and 17 years. For the establisment of reference values results of 396 dogs in the age of 1-9 years were used. Analysis of haematological and clinical-chemical parameters was performed using the following devices: Cell-Dyn 3500, Hitachi 911, GEM Premier 3000 and the coagulometer BE CL 4. The statistical program SPSS was used to calculate the reference values. Results: In $75 \%$ of the tested parameters no major discrepancies were noted in comparison to existing reference values. For eosinophils, basophils, monocytes, alanine aminotransferase, alkaline phosphatase, glutamate dehydrogenase, lipase, creatine kinase, bilirubin and creatinine clear deviations from the existing reference values could be demonstrated. The reference ranges for eosinophils, monocytes and glutamate dehydrogenase were higher than reported in the literature while the reference values for basophils were lower. The reference ranges for the enzymes alanine aminotransferase, alkaline phosphatase, 
and lipase in young $(<1$ year) and old $\operatorname{dogs}(\geq 10$ years) significantly differed from the reference values of dogs at the age of 1-9 years. Conclusion and clinical relevance: Reference values have to be re-evaluated over time since due to continuing developments and knowledge some factors of the measurement, mostly methods and equipment, change. 


\section{3. $\quad$ Einleitung}

Die Bestimmung hämatologischer und klinisch-chemischer Parameter ist ein wichtiges Hilfsmittel bei der Diagnostik innerer Erkrankungen. Die Untersuchungen erfolgen heutzutage nicht nur in kommerziellen Laboren, sondern vielfach auch in den Tierarztpraxen. Die zunehmende Zahl automatisierter Hämatologiegeräte und Arbeitstechniken macht es erforderlich, die Laborergebnisse mit den zur Verfügung stehenden Referenzwerten aus der Literatur zu vergleichen und gegebenenfalls eine Anpassung vorzunehmen. Nur so liefern diese Geräte verlässliche Befunde.

An einer großen Anzahl klinisch gesunder, adulter Hunde wurden im Labor der Medizinischen Kleintierklinik Ludwig-Maximilians-Universität München mit den dort verfügbaren Geräten hämatologische und klinisch-chemische Parameter untersucht. Ziel der Studie war, entsprechende Referenzbereiche zu erstellen und mit den in der Literatur genannten Referenzwerten zu vergleichen. Ferner sollte überprüft werden, ob Alter, Geschlecht sowie Fütterung der Hunde die Referenzbereiche beeinflussen.

\section{Material und Methoden}

\section{Tiere}

In einem Zeitraum von 12 Monaten wurden Blutproben von insgesamt 533 Hunden gewonnen. Im Zuge einer Ausreißereliminierung wurden die Messergebnisse von 15 Hunden aus der Auswertung genommen. Zur Referenzwertbestimmung dienten die Messwerte der adulten Hunde im Alter von 1-9 Jahren $(n=406)$. Hierdurch sollte eine große Streuung der Referenzwerte vermieden werden, da für einige Blutparameter eine Altersabhängigkeit besteht. Dadurch fielen 60 Tiere unter $1 \mathrm{Jahr}$ und 52 Tiere über 9 Jahre für die Referenzwerterstellung weg. Bei gezielter Prüfung einzelner Grenzwerte der Referenzbereiche ließen sich bei einigen Blutwerten Messfehler nicht ausschließen. Bei drei Hunden wiesen die Laborwerte auf eine mögliche Erkrankung hin und die Tiere wurden nach einer erneuten klinischen Untersuchung aus der Studie eliminiert. Gleiches galt für sieben Hunde, die bei einzelnen Parametern sehr hohe Messwerte aufwiesen und nicht erneut untersucht werden konnten. Der Erstellung der Referenzbereiche lagen somit die Daten von 
396 Hunden zugrunde und die Auswertung erfasste insgesamt 508 Hunde.

Bei den 281 Hündinnen und 227 Rüden handelte es sich um Mischlinge ( $\mathrm{n}=143$ ) sowie Vertreter von 55 verschiedenen Hunderassen $(n=365)$ im Alter von < 1 Jahr bis 17 Jahren (Tab. 1). Die am häufigsten vertretenen Rassen waren Beagle $(\mathrm{n}=44)$, Border Collie $(\mathrm{n}=29)$, Jack Russell Terrier $(\mathrm{n}=23)$, Deutscher Schäferhund ( $\mathrm{n}=22)$, Rhodesian Ridgeback $(\mathrm{n}=19)$, Dackel $(\mathrm{n}=17)$, Golden Retriever $(n=13)$, Berner Sennenhund $(n=11)$, Labrador Retriever $(n=11)$ und Harzer Fuchs $(\mathrm{n}=10)$. In Bezug auf das Alter wurden drei Gruppen gebildet: Gruppe 1: < 1 Jahr $(\mathrm{n}=60)$, Gruppe 2: 1-9 Jahre (medianes Alter 3,76 Jahre; $\mathrm{n}=$ 396), Gruppe 3: $\geq 10$ Jahre $(n=52)$. Untersucht wurden sowohl nüchterne (12 Stunden keine Futteraufnahme) als auch nicht nüchterne Tiere (Tab. 1). Die Hunde stammten aus Privathaushalten $(n=366)$, Zuchtbeständen $(n=43)$, Tierheimen $(n=60)$ sowie aus dem klinikeigenen Labor $(n=39)$. Die Laborhunde hatten an einer Futtermittelstudie teilgenommen.

Vor der Blutentnahme erfolgte bei jedem Hund eine klinische Überprüfung des Gesundheitszustands, wobei darauf geachtet wurde, die Tiere möglichst wenig zu stressen. Untersucht wurden Allgemeinbefinden, Körpertemperatur, Gewicht, adspektorisch Bewegungsapparat, Haut und Haarkleid, Maulhöhle und Schleimhäute, palpatorisch Lymphknoten, Puls, kapillare Füllungszeit, Abdomen sowie auskultatorisch Herz und Lunge. Neben diesen Befunden gingen bisherige medizinische Probleme, Medikamentengabe, Zeitpunkt der letzten Fütterung, Impfstatus und Auslandsaufenthalt in die Dokumentation ein. Von der Studie ausgeschlossen blieben Hunde, bei denen sich Hinweise auf eine Erkrankung ergaben.

\section{Probengewinnung und Messmethoden}

Die Blutprobenentnahme erfolgte mit einer 21-Gauge-Kanüle aus der Vena cephalica nach Rasur und Desinfektion der darüber liegenden Hautfläche. Als Auffanggefäße dienten ein 2-ml-EDTA-Röhrchen (Kalium-EDTA; KABE Labortechnik, Nümbrecht-Elsenroth), ein 4,5-ml-Serumröhrchen (Sarstedt, Nümbrecht), eine mit Lithium-Heparin beschichtete 150- $\mu 1$-Kunststoffkapillare für die Blutgasbestimmung (1,9 x $100 \mathrm{~mm}$; Sanguis Counting $\mathrm{GmbH}$ in Nümbrecht) sowie eine 3-ml-Koagulationsmonovette mit 3,8\%iger wässriger 
Natrium-Citrat-Lösung (Sarstedt, Nümbrecht) für die Analyse der Blutgerinnung.

Die Proben unterlagen einer umgehenden Untersuchung oder wurden (bei Blutentnahme in Zuchtbeständen und Tierheimen) nach sofortiger Kühlung innerhalb von 3 Stunden in der Laboreinrichtung der Medizinischen Kleintierklinik untersucht. Die Blutgasanalyse beschränkte sich auf in der Kleintierklinik gewonnene Proben und fand innerhalb von maximal 3 Minunten nach der Blutgewinnung mit dem Blutgasanalysegerät GEM Premier 3000 (Instrumentation Laboratory $\mathrm{GmbH}$, Kirchheim) statt.

Mit dem Analysegerät Cell-Dyn 3500 wurden aus dem EDTA-Blut (Probe von $100 \mu \mathrm{l}$ ) folgende Parameter bestimmt: Leukozyten, neutrophile, eosinophile und basophile Granulozyten, Lymphozyten, Monozyten, Thrombozyten, MPV, Erythrozyten, Hämoglobin, Hämatokrit, RDW, MCV, $\mathrm{MCH}$ und $\mathrm{MCHC}$. Vergleichend erfolgte eine mikroskopische Bestimmung des Differenzialblutbilds (Anfertigung eines EDTA-Blut-Ausstrichs und Färbung mittels Wright-Färbung [Modified Wright's Stain], Differenzierung von 100 Leukozyten). Die Retikulozyten wurden nach Anfärbung einer Probe mit einer alkoholischen Brilliantkresylblau-Lösung ausgezählt. Zur Ermittlung des Hämatokritwerts diente eine Hettich Tischzentrifuge HAEMATOKRIT 210. Bearbeitung und Auswertung der Blutproben nahmen ein veterinärmedizinisch-technischer Assistent, ein medizinisch-technischer Assistent und ein chemisch-technischer Assistent mit jeweils langjähriger Berufserfahrung vor.

Die mit dem Analysegerät Hitachi 911 bestimmten Parameter sowie die dafür verwendeten Messmethoden sind aus Tab. 2 ersichtlich. Die Untersuchung der Blutgerinnung wurde mit dem Kugelkoagulometer BE CL 4 (Behnk Elektronik, München) durchgeführt (Tab. 2). Zum Einsatz kam ein optimierter/modifizierter aus der Humanmedizin stammender Quick-Test, der bei Tieren mit einer höheren Aktivität der Gerinnungsfaktoren Verwendung findet (1). Durch Vorverdünnung der Probe 1:20 mit Diethylbarbiturat-Azetat-Puffer (DBA-Puffer) und Zugabe einer Fibrinogenlösung wurde eine Empfindlichkeitssteigerung durch Verlängerung der Gerinnung erreicht (1).

Die Messgeräte unterlagen einer regelmäßigen Kalibirierung und Qualitätskontrollen mit Kontrollblut. 


\section{Statistische Auswertung}

Zur Aufbereitung der Daten diente das Statistikprogramm SPSS 14 (SPSS Inc. Headquaters, Chicago, USA). Die Überprüfung der Daten mit dem KolmogorovSmirnov-Test und Lilliefors auf Normalverteilung $(3,7,19,21,28)$ ergab, dass die Daten nicht normal verteilt waren. Nach der Ausreißereliminierung nach Henry et al. (13) gingen die Messergebnisse von 508 Hunden in die Auswertung ein. Selbst wenn nur bei einem einzelnen Parameter ein Ausreißer vorlag, blieben alle Messwerte des entsprechenden Hundes von der Auswertung ausgeschlossen. Um einen ersten Überblick zu erhalten, wurden die wichtigsten Lage- und Streuparameter wie Mittelwert, Standardabweichung, Minimum, Maximum und Median ermittelt $(10,19)$. Aufgrund der fehlenden Normalverteilung wurden die Daten nach ihrer Größe sortiert und in absteigender Rangordnung angezeigt. Die Angabe der Referenzbereiche erfolgte nach Ermittlung des 2,5\%- und 97,5\%Perzentils in Form des im Allgemeinen bevorzugten 95\%-Perzentil-Intervalls (16). Vergleichend wurden die Referenzbereiche als 90\%-Perzentil-Intervall bestimmt.

Der Einfluss von Alter, Geschlecht sowie Futteraufnahme auf die verschiedenen Parameter wurde mit dem Kruskal-Wallis-Test/Bonferoni-Korrektur $(\alpha=0,05 / 100)$ oder/und mit dem Mann-Whitney-Test auf Signifikanz geprüft. Entscheidend für das Testverfahren war die Anzahl der unabhängigen Variablen der einzelnen Faktoren (10).

\section{Ergebnisse}

Die Resultate der Referenzwertbestimmung, durchgeführt für die Altersgruppe 2 (1-9 Jahre, medianes Alter 3,76 Jahre), sind in Tab. 3-7 zusammengefasst. Tab. 3 und Tab. 4 listen die Referenzwerte der hämatologischen bzw. klinischchemischen Parameter. Bei $75 \%$ der untersuchten Parameter bestand Übereinstimmung mit den in der Medizinischen Kleintierklinik der LudwigMaximilians-Universität München zugrunde gelegten Referenzbereichen. Ein ähnlicher Prozentsatz war auch im Vergleich mit Referenzwertangaben der Literatur zu verzeichnen. Deutliche Abweichungen zeigten sich bei folgenden Parametern: eosinophile Granulozyten, basophile Granulozyten, Monozyten und Glutamatdehydrogenase, wobei die Referenzbereiche bei eosinophilen 
Granulozyten, Monozyten und GLDH höher lagen, derjenige der basophilen Granulozyten dagegen niedriger, als in der Literatur angegeben. Die Referenzwerte für Alaninaminotransferase, Kreatinkinase, Bilirubin und Kreatinin ließen eine leichte Verschiebung erkennen oder grenzten die Spannweite der Referenzbereiche des Labors weiter ein.

Die Ergebnisse der Prüfung auf eine Abhängigkeit der Referenzwerte von Alter, Geschlecht und Fütterung sind in Tab. 5 und Tab. 6 dargestellt. Bei den Enzymen ALT, AP und Lipase unterschieden sich die Referenzbereiche der jungen und alten Hunde signifikant von den Referenzbereichen der 1-9 Jahre alten Tiere. Weitere Parameter, die bei den jungen Hunden eine signifikante Altersabhängigkeit zeigten, sind in Tab. 5 aufgelistet. Für die Parameter Cholesterin, Triglyzeride und Thrombozyten ergaben sich bei den Hunden im Alter von 10 Jahren und älter signifikant höhere Referenzbereiche. Eine signifikante Geschlechtsabhängigkeit der Referenzbereiche konnte bei den Parametern $\alpha$-Amylase, Lipase, Leukozyten und Lymphozyten festgestellt werden (Tab. 6). Die bei Blutabnahme nüchternen Hunde wiesen signifikant niedrigere Referenzbereiche für Serumgallensäuren und Harnstoff und einen signifikant höheren Referenzbereich für Triglyzeride auf als die gefütterten Probanden (Tab. $6)$.

\section{Diskussion}

Die Referenzwertberechnung erfolgte mit einem 95\%-Perzentil-Intervall und vergleichend mit einem 90\%-Perzentil-Intervall. Da nicht auszuschließen war, dass die Hunde aus dem Tierheim aufgrund der teilweise unbekannten Vorgeschichte zum Zeitpunkt der Blutabnahme eine bei der klinischen Untersuchung nicht erkennbare Erkrankung hatten, wurden vergleichend Referenzwerte ohne Tierheimhunde erstellt. Das Ergebnis zeigte jedoch keine signifikanten Unterschiede.

Die ermittelten Referenzwerte bestätigen die meisten in der Literatur beschriebenen Referenzbereiche (1, 6, 9, 11, 15, 16, 18, 27, 33). Abweichungen von publizierten Referenzwerten $(15,16,26,28,30,33)$ bestanden bei den Parametern eosinophile Granulozyten, basophile Granulozyten und Monozyten, wobei die Diskrepanz im Vergleich zu den Angaben von Kraft und Dürr (16) am 
deutlichsten ausfiel.

Die mit dem Cell-Dyn 3500 ermittelte Zahl der eosinophilen Granulozyten (01,13 G/1 für Hunde von 1-9 Jahren) lag deutlich höher als in der Literatur (0-0,6 G/l) angegeben $(16,31)$. Die manuell erstellten Differenzialblutbilder wiesen ähnlich erhöhte Werte auf (0-1,28 G/l). Neuerer (25) prüfte das Hämatologiegerät Cell-Dyn 3500 auf Zuverlässigkeit bei der Analyse von Hunde- und Katzenblutproben. Er kam zu dem Ergebnis, dass das Gerät aufgrund niedriger Variationskoeffizienten eine hohe Präzision aufweist und stellte auch bei den eosinophilen Granulozyten trotz niedriger absoluter Zellzahlen eine hohe Korrelation zwischen der mikroskopisch und der mit dem Cell-Dyn 3500 bestimmten Zellzahl fest (25). Im Vergleich mit den Referenzwerten der Universität Zürich (0,1-1,16 G/1) (9) und denen des Labors Laupeneck (0,1-1,25 G/l) (18) war nur eine geringfügige Abweichung zu erkennen. Ein direkter Vergleich gestaltet sich schwierig, da die Messmethoden, Populationen und Qualitätskontrollen der anderen Labore nicht bekannt sind.

Bei allen Hunden unterschiedlicher Herkunft ergab die klinische Untersuchung keine Hinweise auf eine Parasiteninfektion oder ein allergisches Geschehen. Eine Kotuntersuchung erfolgte nicht. Möglicherweise hatten einige Hunde eine Parasiteninfektion oder eine Allergie. Bei den Hunden, die zur Blutabnahme in der Medizinischen Kleintierklinik vorgestellt wurden, sowie den Laborhunden war laut Anamnese keine Allergie bekannt. Eine Entwurmung fand nach Angabe der Besitzer und Züchter wenigstens einmal im Jahr statt. Aus der Annahme heraus, dass bei Tierheimhunden aufgrund des Tierverkehrs, ständig wechselnder Tiergruppen, einer unregelmäßigen Entwurmung und oft mangelnder Hygiene eine erhöhte Parasitenbürde mit resultierender Eosinophilie vorliegen könnte, wurden zusätzlich die Referenzwerte der 1-9 Jahre alten Hunde ohne Tiere aus dem Tierheim berechnet (Tab. 3). Auch bei dieser Berechnung stimmte die obere Referenzbereichsgrenze (1,12 G/l) nicht mit den Werten aus der Literatur (16) überein. Kraft und Dürr (16) beschreiben eine Eosinophilie des Deutschen Schäferhundes. Unter den untersuchten Hunden befand sich nur ein Schäferhund mit einer Zellzahl von 1,08 G/l, sodass der höhere Referenzwert nicht mit diesem Krankheitsbild in Zusammenhang stehen kann.

Der ermittelte höhere Referenzbereich erklärt sich möglicherweise durch den 
Klimawandel und die dadurch für Parasiten günstigeren Lebensbedingungen in Deutschland. Forschungen zufolge kann die globale Erderwärmung zu einer Änderung der geographischen Verbreitung von Infektionskrankheiten führen (5). Unter Umständen spielten auch Allergien bei den Hunden eine Rolle. Nicht nur beim Menschen, sondern auch bei den Tieren wird eine Zunahme von Allergien dokumentiert $(2,12,14,22)$.

Im Fall der basophilen Granulozyten gestaltet sich die Referenzbereichserstellung mit dem Gerät Cell-Dyn 3500 schwierig, da diese Zellen nur in sehr geringer Anzahl im Blut vorliegen. Neuerer (25) ermittelte eine nur mäßige Korrelation zwischen manueller und automatisierter Bestimmung und verzichtete daher auf eine statistische Auswertung. Lilliehöök und Tvedten (20) überprüften das Gerät Cell-Dyn 3500 bei Hunden mit einer mikroskopisch nachgewiesener Basophilie und stellten eine Unzuverlässigkeit des Geräts bei der Differenzierung dieser Zellpopulation fest.

Die vom Cell-Dyn 3500 und mittels mikroskopischer Zelldifferenzierung bestimmte obere Referenzbereichsgrenze für die Monozytenzahl betrug 1,29 G/1 bzw. 0,92 G/1. Beide Werte liegen höher als die von Kraft und Dürr (16) (0,5 G/1) sowie Willard und Tvedten (34) (0,43 G/l) angegebenen Werte. Ein nur geringer Unterschied ergab sich beim Vergleich mit dem von Grether (9) genannten Referenzbereich (0,2-0,9 G/1). Weskamp (32) vermutet bei der Monozytenzahl eine Altersabhängigkeit. Eine Korrelation konnte nicht ermittelt werden. Ferner beschreibt sie unterschiedliche Referenzwerte bei verschiedenen Fütterungszeitpunkten (32). In der eigenen Studie ließ sich kein Einfluss der Fütterung nachweisen.

Die Glutamatdehydrogenase (GLDH) reagiert bei Lebererkrankungen sehr empfindlich mit einer Aktivitätssteigerung (16). In der vorliegenden Studie ergab sich bei Hunden unter einem Jahr ein signifikant niedrigerer Referenzbereich (210 U/l). Möglicherweise ist die Leber bei adulten Tieren stärker belastet oder geschädigt als bei jungen Hunden. Eine GLDH-Aktivität über dem Dreifachen der oberen Referenzbereichsgrenze deutet auf eine Leberkrankheit mit Zellnekrose hin, während einer kurzzeitigen Erhöhung bis $15 \mathrm{U} / \mathrm{l}$ nicht pathologisch sein muss. Zudem stellten Kraft und Dürr (16) aufgrund isoliert höherer Werte ohne Nachweis einer Lebererkrankung die Aussagefähigkeit dieses Parameters bei 
Haustieren infrage. Der von uns ermittelte Referenzbereich von 1-12 U/l liegt mit der oberen Grenze doppelt so hoch wie der von Kraft und Dürr (16) angegebene (bis $6 \mathrm{U} / \mathrm{l}$ ).

Für die Serumaktivität der alkalischen Phosphatase (AP) ergab sich der Referenzbereich mit 13-134 U/1. Durch Nutzung verschiedener Puffer bei der Analytik variieren die Referenzwertangaben bei diesem Enzym besonders stark (16). Der Kruskal-Wallis-Test sowie der Mann-Whitney-Test ergaben hoch signifikante $(\mathrm{p}<0,0001)$ Unterschiede in der AP-Aktivität zwischen den Hunden mit einem Alter $<1$ Jahr und den Hunden zwischen 1 und 9 Jahren sowie zwischen den Hunden der Altersgruppe 1-9 Jahre und $\geq 10$ Jahre. Damit bestätigt sich die Aussage von Dereser (6), dass die AP-Aktivität in Abhängigkeit vom Alter signifikant differiert (14-108 U/l für Hunde von 1-8 Jahre und 17-357 U/1 für Hunde aller Altersgruppen). Kuhl et al. (17) stellten ebenfalls eine hohe Aktivität der AP bei jungen Hunden fest, die den Referenzbereich der adulten Hunde beträchtlich überstieg.

Die Referenzwerte für die Lipase differieren in der Literatur stark. Die obere Referenzbereichsgrenze wird mit 300 U/l (16), 527 U/l (34) und 801 U/1 (6) angegeben. Nach Kraft und Dürr (16) können hohe Lipasewerte vorkommen, weshalb die Autoren erst Werte, die die obere Referenzbereichsgrenze mehr als dreifach überschreiten, als aussagekräftig betrachten (16). In der vorliegenden Studie lag der Referenzbereich bei 74-563 U/l. Ältere Hunde wiesen einen signifikant höheren Referenzbereich auf (Tab. 5). Ferner wurde bei Rüden ein signifikant geringerer Referenzbereich ermittelt als bei Hündinnen (Tab. 6). In der vorliegenden Literatur fanden sich dazu keine Angaben.

Der Referenzbereich der Triglyzeride lag mit 0,3-2,8 mmol/l niedriger als in der Literatur angegeben $(0,29-3,88 \mathrm{mmol} / \mathrm{l}$ [16], 0,1-5,7 mmol/1 [34]). Auch bei diesem Parameter bestand eine signifikante Altersabhängigkeit mit deutlich höheren Werten bei den Hunden im Alter von $\geq 10$ Jahren (Tab. 5).

Die Konzentration der Serumgallensäuren hängt stark vom Zeitpunkt der letzten Nahrungsaufnahme ab. Hier sollte nur der Referenzwert der nüchtern getesteten Hunde berücksichtigt werden (Tab. 3, Tab. 6). Er wurde mit 0-18 $\mu \mathrm{mol} / \mathrm{l}$ ermittelt und bestätigt den Referenzbereich von Kraft und Dürr (16). Schlesinger (29) 
nennt einen sehr tiefen präprandialen Referenzwert von $<10 \mu \mathrm{mol} / \mathrm{l}$ und einen postprandialen von $<20 \mu \mathrm{mol} / \mathrm{l}$. Willard und Tvedten (34) unterteilen den Referenzbereich: nüchtern $(0-15,3 \mu \mathrm{mol} / \mathrm{l})$ und 2 Stunden postprandial $(0-20,3$ $\mu \mathrm{mol} / \mathrm{l})$. Das Labor Laupeneck (18) gibt einen postprandialen Wert bis $30 \mu \mathrm{mol} / \mathrm{l}$ an. Hier ist unklar, wann nach der Fütterung die Messung erfolgte. Nach Kraft und Dürr (16) beträgt der postprandiale obere Referenzwert $40 \mu \mathrm{mol} / \mathrm{l}$.

Die festgestellten oberen Referenzbereichsgrenzen von ALT (96 U/l), Bilirubin $(5,5 \mu \mathrm{mol} / \mathrm{l})$, Kreatinkinase $(\mathrm{CK} ; 268 \mathrm{U} / \mathrm{l})$ und Kreatinin $(119 \mu \mathrm{mol} / \mathrm{l})$ liegen höher als die entsprechenden Werte von Kraft und Dürr (16), aber unter denen von Willard und Tvedten (34) (Bilirubin 10,2 $\mu$ mol/l, CK 529 U/l, Kreatinin 124 $\mu \mathrm{mol} / \mathrm{l})$.

Für die aktivierte partielle Thromboplastinzeit (aPTT) wurde ein Referenzbereich von 9,6-14,9 s ermittelt. Mischke und Nolte (23) testeten verschiedene Reagenzien unterschiedlicher Hersteller und bestimmten Referenzwerte für die Gerinnung. Mit dem auch von uns verwendeten PTT-Reagens von Roche (Roche, Mannheim) ergab sich ein aPTT-Referenzbereich von 10,5-13,5 s. Der obere Referenzwert kann nicht bestätigt werden. Ebenso wenig stimmt das Ergebnis dieser Studie mit den Referenzbereichen von Regel (26) und Geffré et al. (8) überein (10-13 s bzw. 12,9-17,3 s). Einen vergleichbaren Referenzwert ermittelten Bauer et al. (4) mit 10,0-14,3 s.

Bei der Bestimmung der Thrombinzeit kam das gleiche Testreagens (STAThrombin, Roche, Mannheim) zum Einsatz wie in der Studie von Mischke (24) sowie von Regel (26). Der von uns bestimmte Referenzbereich betrug 8,23-16,76 s, der von Mischke (24) 14-18 s. Hier lässt sich der obere Referenzwert bestätigen, während umgekehrt der untere Referenzwert mit dem von Regel (27) angegebenen Referenzbereich von 9-12 s übereinstimmt.

Sowohl bei der aPTT wie auch der Thrombinzeit hängen die bestimmten Werte von dem verwendeten Testreagens, zum Teil auch von den Reagenschargen ab. Weitere Einflussfaktoren sind die angewendete Methode, bei der Thrombinzeit auch die Ionenstärke, Thrombinaktivität und die im Testansatz vorhandene Plasmakonzentration (16). Die hier angegebenen Referenzwerte können bei Verwendung anderer Testansätze oder Reagenzien nur als Orientierungshilfe 
dienen, da es eine große Auswahl verschiedener Tests und Reagenzien gibt, die nicht miteinander vergleichbar sind und jede Testmethode ihre eigenen Referenzbereiche benötigt (34).

Der Referenzbereich des optimierten Quick-Tests betrug 9,5-22,0 s. Regel (26) bestimmte unter Verwendung eines nicht mehr erhältlichen DiethylbarbituratAzetat-Puffers einen Referenzbereich von 16-27 s, was für die differierenden Referenzbereiche verantwortlich sein kann.

\section{Fazit}

Die Bestimmung von Referenzwerten ist in regelmäßigen Abständen, besonders bei neuen Methoden und Geräten, sinnvoll und notwendig. Diese Studie zeigt erneut, dass eine nicht geprüfte Übernahme von Referenzwerten aus anderen Laboren oder der Literatur mit unbekannten Messmethoden nicht verlässlich ist. Teilweise konnten bestehende Referenzwerte bestätigt werden oder es ergaben sich nur minimale Abweichungen. Bei einigen Parametern ließ sich eine Abhängigkeit von Geschlecht und Alter und letzter Fütterung der Hunde nachweisen, die nicht in allen Fällen in der vorliegenden Literatur dokumentiert wurde.

Interessenkonflikt

Die Autoren bestätigen, dass kein Interessenkonflikt besteht.

\section{Literaturverzeichnis}

1. Abbrederis N. Validierung von Laborparametern zur Diagnose einer DIC beim Hund. Diss. med. vet. Ludwig Maximilian University Munich 2005.

2. Asher I. Worldwide time in the prevalence of symptoms of asthma, allergic rhinoconjunctivitis, and eczema in childhood. ISAAC Phases One and Three repeat multicountry cross-sectional surveysm, Lancet 2006; 368:733.

3. Backhaus K, Erichson B, Plinke W, Weiber R. Multivariate Analysemethoden. $8^{\text {th }}$ ed. Berlin: Springer 1996; XVII-XXIII, 56-57.

4. Bauer N, Eralp O, Moritz A. Reference intervals and method optimization for variables reflecting hypocoagulatory and hypercoagulatory states in dogs using the STA Compact automated analyzer. J Vet Diagn Invest 2009; 21(6), 803-14.

5. Bayrisches Staatsministerium für Umwelt, Gesundheit und Verbraucherschutz [homepage of the Internet]. München: Klima im Wandel - Impulse der 
Umweltbildung; 2009. Bayrisches Staatsministerium für Umwelt, Gesundheit und Verbraucherschutz, 2009 - Publikationen. Available from: http://www.stmug.bayern.de/umwelt/klimaschutz/klimawandel/index.htm

6. Dereser R. Blutchemische Referenzbereiche in der Labordiagnostik des Hundes. Diss. med. vet. Ludwig Maximilian University Munich 1989.

7. Eimer E. Varianzanalyse, 1st ed., Stuttgart, Berlin, Köln, Mainz: Kohlhammer Verlag 1978; 7.

8. Geffré A, Grollier S, Hanot C, Vergez F, Trummel C, Braun JP. Canine reference intervals for coagulation markers using the STA Satellite(R) and the STA-R Evolution(R) analyzers. J Vet Diagn Invest 2010; 22(5), 690-5.

9. Grether, B., 2008: Veterinärmedizinisches Labor der Universität Zürich. Vet.uzh. [homepage of the Internet]. Available from: http://www.vet.uzh.ch/Studium/vetmed/studiengang/jk5/Jk5_rotation_blockb_200 7_8.pdf

10. Guggenmoos-Holzmann I, Wernecke D. Medizinische Statistik, 1st ed.

Berlin: Blackwell Wissenschaftsverlag 1995; 93-97.

11. Hartmann, K, Referenzbereiche in der Labordiagnostik der Katze. Diss. med. vet. Ludwig-Maximilians-University Munich 1990.

12. Heil-Franke G, Hunsinger B. Spezifische Immuntherapie (SIT) beim Hund: Einfluss von Tierhalter, Art und Anzahl der Allergene. Kleintierpraxis 2002; 47, 483-487.

13. Henry R J, Cannon DC, Winkelman JW. Normal values and the use of laboratory results for the detection of disease. Clinical Chemistry - Priniciples and Technics. $2^{\text {nd }}$ ed. New York: Harper\&Row 1974; 343-71.

14. Hermann-Kunz E. Allergische Krankheiten in Deutschland Ergebnisse einer repräsentativen Studie. Bundesgesundheitsbl. - Gesundheitsforsch. Gesundheitsschutz 2000; 43, 400-406.

15. Kraft H, Schillinger D, Klinische Labormethoden der Veterinärmedizin bei Haussäugetieren, $3^{\text {rd }}$ ed. Stuttgart: Enke 1989; 5,228-231.

16. Kraft W, Dürr UM.: Klinische Labordiagnostik in der Tiermedizin. $6^{\text {th }}$ ed. Stuttgart: Schattauer 2005; 2-4,15,85,126,130-134,141,152,170,507-523.

17. Kuhl S, Mischke R, Lund C, Günzel-Apel A.-R. Referenzwerte klinischchemischer Blutparameter bei Hundewelpen in den ersten acht Lebenswochen. Dtsch. Tierärztl. Wschr 2000; 107, 429-468.

18. Laupeneck, 2008: Laupeneck [hompage of the Internet]. Bern: Referenzwerte für Hund und Katze; [updatet 24.04.2008; cited 14.05.2008]. Available from: http://www.labor-laupeneck.ch/d/dienstl/pdf/d_normalwerte.pdf

19. Laufner W. Beschreibende Statistik für Betriebswirte. 1st ed. Dortmund: Fachhochschule Dortmund 2000; Modul 03:2, 79.

20. Lilliehöök I, Tvedten HW. Errors in basophil enumeration with 3 veterinary hematology systems and observations on occurrence of basophils in dogs. ASVCP 
2011; 40(4):450-8

21. Meffert H. Marktforschung. $2^{\text {nd }}$ ed. Wiesbaden: Gabler 1992; 300.

22. Meyer R.. Zunahme von Allergien bei Kindern. Kinderumweltgesundheit [homepage of the Internet]. Köln: Deutsches Ärzteblatt 2008; Available from: http://www.kinderumweltgesundheit.de/KUG/index2/pdf/dokumente/50053_1

23. Mischke R, Nolte I. Haemostasis diagnostics in dogs: 2. Principle, technique and reference ranges of different tests. Prakt Tierarzt 1999; 80(10), 836-55.

24. Mischke R. Optimization of coagulometric tests that incorporate human plasma for determination of coagulation factor activities in canine plasma. Am $\mathbf{J}$ Vet Res 2001; 62, 625-629.

25. Neurer F. Evaluation des vollautomatischen Hämatologiegerätes Cell-Dyn 3500 im klinischen

Einsatz bei Hund und Katze. Diss. med. vet. Ludwig Maximilian University Munich 2005.

26. Regel A. Die disseminierte intravasale Gerinnung beim Hund. Diss. med. vet. Ludwig-Maximilian University Munich 2002.

27. Sassnau S. Veränderungen des Blutbildes bei Siberian Huskys in Abhängigkeit vom Trainingszustand. Tierärztl Prax 2007; 35, 375-380.

28. Schach S, Schäfer T. Regressions und Varianzanalyse. $1^{\text {st }}$ ed. Berlin, Heidelberg, New York: Springer 1978; 172ff.

29. Schlesinger DP, Rubin SI. Serum bile acids and the assessment of hepatic function in dogs and cats. Can Vet J Volume 1993; 34, 215-219.

30. Siegling-Vlitakis C, Kohn B, Kellermeier C, Schmitz R, Hartmann H.

Eignung der Stewart-Variablen zur Beurteilung des Säuren-Basen-Status bei gesunden und unterschiedlich erkrankten Hunden. Berl Münch Tierärztl Wochenschr 2007; 120(3/4), 148-155.

31. Ved Med Labor GmbH. Leistungsverzeichnis IDEXX Vet Med Labor, 1. Neuauflage Ludwigsburg: 2007; Available from: www.vetmedlabor.de/downloads/VetMedLabor_LVZ_02-2007.pdf

32. Weskamp M. Referenzbereiche in der Labordiagnostik des Hundes: Glucose, Harnstoff, Cholesterin, Triglyceride und Hämatologie. Diss. med. vet. LudwigMaximilians-University Munich 1993.

33. Wiinberg B, Lundorff Jensen A, Kjelgaard-Hansen M, Rojkjaer R, Johansson PI, Gade LP, et al. Study on biological variation of haemostatic parameters in clinically healthy dogs. The Veterinary Journal 2007; 174, 62-68.

34. Willard MD, Tvedten H. Small Animal Clinical Diagnosis by Laboratory Methods. $4^{\text {th }}$ ed. Philadelphia: Imprint of Elsevier Science Limited 2004; 125,533537. 


\section{Tabellen}

Tab. 1 Angaben zu den Hunden $(\mathrm{n}=508)$

Table 1 Information on the dogs $(n=508)$.

\begin{tabular}{|l|l|l|}
\hline Parameter & Hunde (n) \\
\hline Alter (Jahre) & $<1$ & 60 \\
\cline { 2 - 3 } & $1-9$ & 396 \\
\cline { 2 - 3 } & $10-17$ & 52 \\
\hline \multirow{2}{*}{ Geschlecht } & männlich & 227 \\
\cline { 2 - 3 } & weiblich & 281 \\
\hline \multirow{2}{*}{ Rasse } & Mischlinge & 143 \\
\cline { 2 - 3 } & Rassehunde & 365 \\
\hline Fütterungszustand & nüchtern * & 140 \\
\cline { 2 - 3 } & nicht nüchtern & 368 \\
\hline * Letzte Futteraufnahme vor 12 Stunden \\
\hline
\end{tabular}


Tab. 2 Analysemethoden der mit den Geräten Hitachi 911 und Coagulometer BE CL 4 bestimmten Parameter (Literatur zur Tabelle beim Verfasser)

Table 2 Analysis methods of parameters that were measured with the Hitachi 911 and the Coagulometer BE CL 4 (references available from the authors).

\begin{tabular}{|c|c|c|}
\hline Gerät & Parameter & Methode \\
\hline $\begin{array}{l}\text { Hitachi } 911 \\
\text { (Roche, } \\
\text { Mannheim) }\end{array}$ & Aspartataminotransferase & Standard-UV-Test \\
\hline & $\begin{array}{l}\text { alkalische Phosphatase (ALP; } \\
\text { E.C. 3.1.3.1), "erhitzte alkalische } \\
\text { Phosphatase" (Serum auf } 65^{\circ} \mathrm{C} \\
\text { erhitzt) }\end{array}$ & Standard-Farbtest \\
\hline & Glutamatdehydrogenase & Standard-UV-Test \\
\hline & y-Glutamyl-Transferase & enzymatischer Farbtest \\
\hline & $\begin{array}{l}\alpha \text {-Amylase (1,4 } \alpha \text {-D-Glucano- } \\
\text { hydrolasen, EC 3.2.1.1) }\end{array}$ & $\begin{array}{l}\text { enzymatischer Farbtest gemäß International } \\
\text { Federation of Clinical Chemistry }\end{array}$ \\
\hline & Lipase & enzymatischer Farbtest \\
\hline & Kreatinkinase & $\begin{array}{l}\text { UV-Test mit Imidazol- und 3-(Cyclohexylamin)- } \\
\text { 2-hydroxy-1-propansulfonsäure-Puffer }\end{array}$ \\
\hline & Bilirubin und Triglyzeride & Farbtest und enzymatischer Farbtest \\
\hline & Cholesterin & $\begin{array}{l}\text { enzymatischer Farbtest mit Piperazin-1,4-bis(2- } \\
\text { ethansulfonsäure)-Puffer }\end{array}$ \\
\hline & Protein & $\begin{array}{l}\text { Farbtest mit Natronlauge und Kaliumjodid- } \\
\text { Reagenz }\end{array}$ \\
\hline & Albumin & $\begin{array}{l}\text { Farbtest mit Endpunkt-Methode und } \\
\text { Zitratpruffer }\end{array}$ \\
\hline & Harnstoff & kinetischer UV-Test \\
\hline & Kreatinin & enzymatischer Farbtest \\
\hline & Glukose & $\begin{array}{l}\text { UV-Test Hexokinasemethode mit Tris(hydroxy- } \\
\text { methyl)-Aminomethan- und 2-(4-(2-Hydroxy- } \\
\text { ethyl)-1-piperazinyl)-ethansulfonsäure-Puffer } \\
\text { als Reagenz }\end{array}$ \\
\hline & Fruktosamin & Farbtest mit Nitrotetrazolium-Blau-Reagenz \\
\hline & Kalzium und Phosphor & Endpunkt-Farbtest und Probenleerwert \\
\hline & Kalium & ionenselektive Elektrode (ISE) \\
\hline & Natrium & ionenselektive Elektrode (ISE) \\
\hline & Chlorid & ionenselektive Elektrode (ISE) \\
\hline Kugelkoagulo- & Thrombinzeit & STA-Thrombin (Roche, Mannheim) \\
\hline $\begin{array}{l}\text { meter BE CL } 4 \\
\text { (Behnk }\end{array}$ & $\begin{array}{l}\text { aktivierte partielle } \\
\text { Thromboplastinzeit }\end{array}$ & PTT-Reagens (Roche, Mannheim) \\
\hline $\begin{array}{l}\text { Elektronik, } \\
\text { München) }\end{array}$ & $\begin{array}{l}\text { optimierter Quick-Test } \\
\text { (Prothrombinzeit) }\end{array}$ & Neoplastin Plus (Roche, Mannheim) \\
\hline
\end{tabular}

Tab. 3 Hämatologische Referenzwerte, bestimmt als 95\%- und 90\%-PerzentilIntervall bei 396 Hunden im Alter von 1-9 Jahren (medianes Alter 3,76 Jahre) sowie bei diesem Probandenkollektiv unter Ausschluss der im Tierheim lebenden Hunde $(\mathrm{n}=350)$. 
Table 3 Haematological reference values, calculated as 95\%- and 90\%-percentile interval in 396 dogs aged 1-9 years (median age 3,76 years) and in the same collective of probands with the exclusion of dogs living in animal shelters $(\mathrm{n}=$ $350)$.

\begin{tabular}{|c|c|c|c|c|c|}
\hline \multirow[t]{2}{*}{ Parameter } & \multirow[t]{2}{*}{$\begin{array}{l}\text { Maß- } \\
\text { einheit }\end{array}$} & \multicolumn{2}{|c|}{$\begin{array}{l}\text { Referenzbereich } \\
(\mathrm{n}=396)\end{array}$} & \multicolumn{2}{|c|}{$\begin{array}{l}\text { Referenzbereich }(n=350 ; \\
\text { ohne Tierheimhunde) }\end{array}$} \\
\hline & & $\begin{array}{l}\text { 95\%-Perzentil- } \\
\text { Intervall }\end{array}$ & $\begin{array}{l}\text { 90\%-Perzentil- } \\
\text { Intervall }\end{array}$ & $\begin{array}{l}\text { 95\%-Perzentil- } \\
\text { Intervall }\end{array}$ & \begin{tabular}{|l|}
$90 \%-$ \\
Perzentil- \\
Intervall
\end{tabular} \\
\hline \multicolumn{6}{|l|}{ Blutbild $^{1}$} \\
\hline Leukozyten & $\mathrm{G} / \mathrm{l}$ & $4,4-15,4$ & $5,0-14,3$ & $4,5-15,4$ & $5,0-14,3$ \\
\hline \multirow[t]{2}{*}{ neutrophile Granulozyten } & $\mathrm{G} / \mathrm{I}$ & $2,96-13,15$ & $3,34-11,00$ & $2,96-13,80$ & $3,32-11,00$ \\
\hline & $\%$ & $47,90-89,60$ & $51,40-85,90$ & $48,67-89,32$ & $52,87-84,43$ \\
\hline \multirow{2}{*}{$\begin{array}{l}\text { eosinophile } \\
\text { Granulozyten }\end{array}$} & $\mathrm{G} / \mathrm{l}$ & $0-1,13$ & $0-0,98$ & $0-1,12$ & $0-0,98$ \\
\hline & $\%$ & $0-10,86$ & $0-10,00$ & $0-10,88$ & $0-10,00$ \\
\hline \multirow{2}{*}{ basophile Granulozyten } & $\mathrm{G} / \mathrm{l}$ & $0-0,25$ & $0-0,19$ & $0-0,23$ & $0-0,18$ \\
\hline & $\%$ & $0-2,19$ & $0-1,79$ & $0-2,12$ & $0-1,74$ \\
\hline \multirow[t]{2}{*}{ Lymphozyten } & $\mathrm{G} / \mathrm{l}$ & $0,54-3,57$ & $0,63-3,21$ & $0,62-3,59$ & $0,72-3,19$ \\
\hline & $\%$ & $4,69-36,66$ & $7,33-34,90$ & $6,28-36,68$ & $8,50-35,02$ \\
\hline \multirow[t]{2}{*}{ Monozyten } & $\mathrm{G} / \mathrm{l}$ & $0,05-1,29$ & $0,07-1,13$ & $0,05-1,20$ & $0,06-1,08$ \\
\hline & $\%$ & $0,59-13,30$ & $0,76-11,95$ & $0,58-12,84$ & $0,75-11,66$ \\
\hline \multirow[t]{2}{*}{$\begin{array}{l}\text { seg. neutrophile } \\
\text { Granulozyten }^{2}\end{array}$} & $\mathrm{G} / \mathrm{l}$ & $1,75-10,71$ & $2,91-10,13$ & $1,78-10,76$ & $2,90-10,15$ \\
\hline & $\%$ & $38,0-84,0$ & $44,0-83,0$ & $37,9-85,0$ & $43,0-83,0$ \\
\hline \multirow[t]{2}{*}{$\begin{array}{l}\text { stab. neutrophile } \\
\text { Granulozyten }^{2}\end{array}$} & $\mathrm{G} / \mathrm{l}$ & $0-0,5$ & $0-0,4$ & $0-0,6$ & $0-0,4$ \\
\hline & $\%$ & $0-5$ & $0-4$ & $0-5$ & $0-4$ \\
\hline \multirow[t]{2}{*}{$\begin{array}{l}\text { eosinophile } \\
\text { Granulozyten }^{2}\end{array}$} & $\mathrm{G} / \mathrm{l}$ & $0-1,28$ & $0-1,06$ & $0-1,22$ & $0-1,04$ \\
\hline & $\%$ & $0-12,00$ & $0-11,00$ & $0-11,00$ & $0-12,00$ \\
\hline \multirow[t]{2}{*}{ basophile Granulozyten ${ }^{2}$} & $\mathrm{G} / \mathrm{l}$ & $0-0,13$ & $0-0,09$ & $0-0,11$ & $0-0,09$ \\
\hline & $\%$ & $0-1,00$ & $0-1,00$ & $0-1,00$ & $0-1,00$ \\
\hline \multirow[t]{2}{*}{ Lymphozyten $^{2}$} & $\mathrm{G} / \mathrm{l}$ & $0,49-4,43$ & $0,71-4,06$ & $0,51-4,43$ & $0,75-4,03$ \\
\hline & $\%$ & $6,50-48,00$ & $8,00-42,95$ & $6,48-48,55$ & $8,00-44,05$ \\
\hline \multirow[t]{2}{*}{ Monozyten $^{2}$} & $\mathrm{G} / \mathrm{l}$ & $0-0,92$ & $0-0,83$ & $0-0,92$ & $0-0,78$ \\
\hline & $\%$ & $0-9,67$ & $0-8,00$ & $0-9,00$ & $0-8,00$ \\
\hline Erythrozyten & $T / /$ & $5,5-9,4$ & $5,8-8,7$ & $5,6-9,5$ & $5,8-8,9$ \\
\hline Hämoglobin & $\mathrm{mmol} / \mathrm{I}$ & $8,2-13,0$ & $8,7-12,6$ & $8,3-13,0$ & $8,8-12,7$ \\
\hline Hämatokrit & $\mathrm{I} / \mathrm{I}$ & $0,37-0,60$ & $0,38-0,58$ & $0,37-0,60$ & $0,39-0,59$ \\
\hline Hämatokrit $^{2}$ & $\mathrm{D} / \mathrm{I}$ & $0,36-0,60$ & $0,39-0,58$ & $0,36-0,60$ & $0,39-0,58$ \\
\hline RDW & $\% c v$ & $13,90-18,51$ & $14,30-18,20$ & $13,90-18,40$ & $14,26-18,09$ \\
\hline MCV & $\mathrm{fl}$ & $61-73$ & $62-72$ & $62-74$ & $63-73$ \\
\hline $\mathrm{MCH}$ & $\mathrm{fmol} / \mathrm{l}$ & $1,3-1,6$ & $1,3-1,5$ & $1,4-1,6$ & $1,4-1,6$ \\
\hline $\mathrm{MCHC}$ & $\mathrm{mmol} / \mathrm{l}$ & $20-23$ & $20-23$ & $21-24$ & $21-23$ \\
\hline Retikulozyten relativ ${ }^{2}$ & $\%$ & $0-0,014$ & $0-0,013$ & $0-0,014$ & $0-0,013$ \\
\hline Retikulozyten absolut ${ }^{2}$ & $\mathrm{G} / \mathrm{l}$ & $0-112$ & $0-99$ & $0-113$ & $0-100$ \\
\hline Thrombozyten & $\mathrm{G} / \mathrm{l}$ & $153-582$ & $176-510$ & $157-568$ & $188-511$ \\
\hline MPV & $\mathrm{fl}$ & $6,75-18,52$ & $7,10-15,70$ & $6,74-17,75$ & $7,07-15,70$ \\
\hline
\end{tabular}


Tab. 3 (Fortsetzung)

\begin{tabular}{|c|c|c|c|c|c|}
\hline \multicolumn{6}{|l|}{ Blutgasanalyse $^{3}$} \\
\hline $\mathrm{pH}-$ Wert & & & & $7,29-7,42$ & $7,31-7,42$ \\
\hline $\mathrm{PCO}_{2}$ & $\mathrm{mmHg}$ & & & $32-54$ & $32-50$ \\
\hline Bikarbonat & $\mathrm{mmol} / \mathrm{l}$ & & & $19-27$ & $19-27$ \\
\hline $\mathrm{TCO}_{2}$ & $\mathrm{mmol} / \mathrm{l}$ & & & $20-29$ & $20-28$ \\
\hline Basenexzess & $\mathrm{mmol} / \mathrm{l}$ & & & $-5,6$ bis 2,5 & $-5,0$ bis 2,1 \\
\hline Glukose & $\mathrm{mmol} / \mathrm{l}$ & & & $3,8-6,4$ & $4,1-6,3$ \\
\hline Laktat & $\mathrm{mmol} / \mathrm{l}$ & & & $0,48-3,13$ & $0,60-2,41$ \\
\hline ionisiertes Kalzium & $\mathrm{mmol} / \mathrm{l}$ & & & $1,2-1,4$ & $1,2-1,4$ \\
\hline Kalium & $\mathrm{mmol} / \mathrm{l}$ & & & $3,3-4,7$ & $3,5-4,6$ \\
\hline \multicolumn{6}{|l|}{ Gerinnungsanalyse ${ }^{4}$} \\
\hline optimierter Quick-Test & $\mathrm{s}$ & $9,5-21,9$ & $10,9-21,0$ & $9,2-22,0$ & $11,0-21,2$ \\
\hline aPTT & $\mathrm{S}$ & $9,6-14,9$ & $9,8-13,4$ & $9,6-15,0$ & $9,8-13,4$ \\
\hline Thrombinzeit & $\mathrm{s}$ & $8,2-16,7$ & $8,9-15,0$ & $8,1-17,0$ & $9,0-15,7$ \\
\hline
\end{tabular}


Tab. 4 Klinisch-chemische Referenzwerte, bestimmt als 95\%- und 90\%-PerzentilIntervall bei 396 Hunden im Alter von 1-9 Jahren (medianes Alter 3,76 Jahre) sowie bei diesem Probandenkollektiv unter Ausschluss der im Tierheim lebenden Hunde ( $\mathrm{n}=350)$.

Table 4 Clinical chemical reference values, calculated as 95\%- and 90\%percentile interval in 396 dogs aged 1-9 years (median age 3,76 years) and in the same collective of probands with the exclusion of dogs living in animal shelters (n $=350)$.

\begin{tabular}{|c|c|c|c|c|c|}
\hline \multirow[t]{2}{*}{ Parameter ${ }^{1}$} & \multirow[t]{2}{*}{$\begin{array}{l}\text { Maß- } \\
\text { einheit }\end{array}$} & \multicolumn{2}{|c|}{$\begin{array}{l}\text { Referenzbereich } \\
(\mathrm{n}=396)\end{array}$} & \multicolumn{2}{|c|}{$\begin{array}{l}\text { Referenzbereich }(n=350 \\
\text { ohne Tierheimhunde) }\end{array}$} \\
\hline & & $\begin{array}{l}\text { 95\%-Perzentil- } \\
\text { Intervall }\end{array}$ & $\begin{array}{l}\text { 90\%-Perzentil- } \\
\text { Intervall }\end{array}$ & $\begin{array}{l}\text { 95\%-Perzentil- } \\
\text { Intervall }\end{array}$ & $\begin{array}{l}\text { 90\%-Perzentil- } \\
\text { Intervall }\end{array}$ \\
\hline AST & $\mathrm{U} / \mathrm{I}$ & $18-52$ & $20-47$ & $18-52$ & $20-47$ \\
\hline$\overline{A L T}$ & $\mathrm{U} / \mathrm{I}$ & $18-96$ & $20-72$ & $18-97$ & $22-72$ \\
\hline $\mathrm{AP}$ & $\mathrm{U} / \mathrm{I}$ & 13-134 & $16-107$ & $13-134$ & $16-109$ \\
\hline $\mathrm{APh}$ & $\mathrm{U} / \mathrm{I}$ & $0-95$ & $0-88$ & $0-93$ & $0-83$ \\
\hline GLDH & $\mathrm{U} / \mathrm{I}$ & $1-12$ & $1-9$ & $1-12$ & $1-9$ \\
\hline $\mathrm{Y}$-GT & $\mathrm{U} / \mathrm{I}$ & $0-8$ & $1-8$ & $1-9$ & $1-8$ \\
\hline$\alpha$-Amylase & $\mathrm{U} / \mathrm{I}$ & $346-1323$ & 386-1124 & $346-1342$ & $386-1120$ \\
\hline Lipase & $\mathrm{U} / \mathrm{I}$ & $74-563$ & 84-477 & $73-553$ & 84-479 \\
\hline Kreatinkinase & $\mathrm{U} / \mathrm{I}$ & $54-268$ & 63-239 & $53-254$ & $61-238$ \\
\hline Bilirubin & $\mu \mathrm{mol} / / \mathrm{l}$ & $0,1-5,5$ & $0,1-3,7$ & $0,1-4,8$ & $0,1-3,8$ \\
\hline Serumgallensäuren ${ }^{2}$ & $\mu \mathrm{mol} / \mathrm{I}$ & $0-18$ & $0-14$ & $0-25$ & $0-17$ \\
\hline Cholesterin & $\mathrm{mmol} / \mathrm{l}$ & $3,5-9,5$ & $3,8-8,9$ & $3,5-9,6$ & $3,8-9,0$ \\
\hline Triglyzeride & $\mathrm{mmol} / \mathrm{l}$ & $0,3-2,8$ & $0,3-2,2$ & $0,3-2,6$ & $0,4-2,1$ \\
\hline Protein & $g / l$ & $55-77$ & $56-74$ & $55-75$ & $57-74$ \\
\hline Albumin & $g / l$ & $31-42$ & $32-41$ & $32-43$ & $33-42$ \\
\hline Globuline & $g / l$ & $19,0-41,8$ & $20,3-37,1$ & $19,0-39,8$ & $20,3-34,7$ \\
\hline Harnstoff & $\mathrm{mmol} / \mathrm{l}$ & $3,5-10,7$ & $4,0-9,6$ & $3,7-10,8$ & $4,2-9,8$ \\
\hline Kreatinin & $\mu \mathrm{mol} / \mathrm{l}$ & $44-119$ & $51-113$ & $48-119$ & $55-116$ \\
\hline Glukose & $\mathrm{mmol} / \mathrm{l}$ & $3,7-6,5$ & $4,0-6,3$ & $3,7-6,6$ & $4,0-6,3$ \\
\hline Fruktosamin & $\mu \mathrm{mol} / \mathrm{l}$ & $207-378$ & $223-353$ & $207-382$ & $224-360$ \\
\hline Kalzium & $\mathrm{mmol} / \mathrm{l}$ & $2,2-2,8$ & $2,3-2,8$ & $2,3-2,8$ & $2,4-2,8$ \\
\hline Phosphor & $\mathrm{mmol} / \mathrm{l}$ & $0,8-2,0$ & $0,9-1,8$ & $0,9-2,0$ & $0,9-1,8$ \\
\hline Natrium & $\mathrm{mmol} / \mathrm{l}$ & $139-162$ & $141-158$ & $139-164$ & $141-158$ \\
\hline Kalium & $\mathrm{mmol} / \mathrm{l}$ & $3,8-5,5$ & $3,9-5,3$ & $3,8-5,5$ & $3,9-5,3$ \\
\hline Chlorid & $\mathrm{mmol} / \mathrm{l}$ & \begin{tabular}{|l|}
$105-118$ \\
\end{tabular} & \begin{tabular}{|l|}
$106-117$ \\
\end{tabular} & $105-119$ & $107-117$ \\
\hline
\end{tabular}


Tab. 5 Parameter mit altersabhängigen Referenzbereichen (bestimmt als 95\%Perzentil-Intervall), Darstellung der signifikanten $(\mathrm{p}<0,0001)$ Unterschiede im Vergleich zu den Referenzbereichen der 1-9 Jahre alten Hunde (Reihung der Parameter nach den Bestimmungsmethoden)

Tab. 5 Age dependent parameters (calculated as 95\%-percentile interval), depiction of significant $(\mathrm{p}<0.0001)$ differences in comparison to the reference values of dogs in the age of 1-9 years (order of the parameters according to measurement methods)

\begin{tabular}{|c|c|c|c|}
\hline Parameter & Maßeinheit & $\begin{array}{l}\text { Referenzbereich } \\
\text { Alter }<1 \text { Jahr } \\
(\mathrm{n}=60)\end{array}$ & $\begin{array}{l}\text { Referenzbereich } \\
\text { Alter } \geq 10 \text { Jahre } \\
(\mathrm{n}=52)\end{array}$ \\
\hline Hämatokrit ${ }^{1}$ & $1 / 1$ & $0,31-0,56$ & $x$ \\
\hline \multirow[t]{2}{*}{ Lymphozyten $^{\top}$} & $\mathrm{G} / \mathrm{I}$ & $0,71-8,20$ & $x$ \\
\hline & $\%$ & $5,85-60,53$ & $x$ \\
\hline $\begin{array}{l}\text { segmentkernige neutrophile } \\
\text { Granulozyten }\end{array}$ & $\%$ & $19,00-86,00$ & $x$ \\
\hline Retikulozyten relativ $^{1}$ & & $0-0,019$ & $x$ \\
\hline ionisiertes Kalzium $^{2}$ & $\mathrm{mmol} / \mathrm{l}$ & $1,36-1,56$ & $x$ \\
\hline optimierter Quick-Test $^{3}$ & $\mathrm{mmol} / \mathrm{l}$ & $11,2-27,5$ & $x$ \\
\hline $\mathrm{ALT}^{4}$ & $\mathrm{U} / \mathrm{I}$ & $13-57$ & $23-290$ \\
\hline $\mathrm{AP}^{4}$ & $\mathrm{U} / \mathrm{I}$ & $24-214$ & $9-200$ \\
\hline $\mathrm{GLDH}^{4}$ & $\mathrm{U} / \mathrm{I}$ & $2-10$ & $x$ \\
\hline Lipase $^{4}$ & $\mathrm{U} / \mathrm{I}$ & $51-745$ & $93-1741$ \\
\hline Kreatinkinase $^{4}$ & $\mathrm{U} / \mathrm{I}$ & $105-716$ & $\mathrm{x}$ \\
\hline Cholesterin $^{4}$ & $\mathrm{U} / \mathrm{I}$ & $x$ & $4,1-10,7$ \\
\hline Triglyzeride $^{4}$ & $\mathrm{mmol} / \mathrm{l}$ & $x$ & $0,34-6,05$ \\
\hline Protein $^{4}$ & $g / l$ & $47-82$ & $x$ \\
\hline Albumin $^{4}$ & $g / l$ & $26-38$ & $x$ \\
\hline Kreatinin $^{4}$ & $\mu \mathrm{mol} / \mathrm{l}$ & $28-107$ & $x$ \\
\hline Glukose $^{4}$ & $\mathrm{mmol} / \mathrm{l}$ & $4,4-6,9$ & $\mathrm{x}$ \\
\hline Fruktosamin $^{4}$ & $\mu \mathrm{mol} / \mathrm{l}$ & $87-303$ & $x$ \\
\hline Kalzium $^{4}$ & $\mathrm{mmol} / \mathrm{l}$ & $2,0-2,9$ & $\mathrm{x}$ \\
\hline Phosphor $^{4}$ & $\mathrm{mmol} / \mathrm{l}$ & $1,0-3,2$ & $\mathrm{x}$ \\
\hline Natrium $^{4}$ & $\mathrm{mmol} / \mathrm{l}$ & $137-157$ & $x$ \\
\hline Kalium $^{4}$ & $\mathrm{mmol} / \mathrm{l}$ & $3,8-6,4$ & $x$ \\
\hline Chlorid $^{4}$ & $\mathrm{mmol} / \mathrm{l}$ & $100-117$ & $x$ \\
\hline Leukozyten $^{5}$ & $G / l$ & $6,25-18,84$ & $x$ \\
\hline neutrophile Granulozyten $^{5}$ & $\mathrm{G} / \mathrm{l}$ & $2,97-14,06$ & $x$ \\
\hline Lymphozyten $^{5}$ & $\mathrm{G} / \mathrm{l}$ & $0,50-7,14$ & $\mathrm{x}$ \\
\hline Erythrozyten $^{5}$ & $T / I$ & $4,51-9,84$ & $\mathrm{x}$ \\
\hline Hämoglobin ${ }^{5}$ & $\mathrm{mmol} / \mathrm{l}$ & $6,56-14,04$ & $x$ \\
\hline Hämatokrit $^{5}$ & $\mathrm{I} / \mathrm{I}$ & $0,30-0,68$ & $x$ \\
\hline Thrombozyten 5 & $\mathrm{G} / \mathrm{l}$ & $x$ & $190-733$ \\
\hline \multicolumn{4}{|c|}{$\begin{array}{l}{ }^{1} \text { Differenzialblutbild manuell erstellt; }{ }^{2} \text { gemessen mit dem Gerät GEM Premier } 3000 ;{ }^{3} \text { gemessen mit } \\
\text { Koagulometer; }{ }^{4} \text { gemessen mit Hitachi } 911 ;{ }^{5} \text { gemessen mit Cell-Dyn } 3500 \\
\text { x = keine signifikanten Unterschiede }\end{array}$} \\
\hline
\end{tabular}


Tab. 6 Parameter mit Referenzbereichen (bestimmt als 95\%-Perzentil-Intervall) mit einer Abhängigkeit vom Geschlecht (Rüden: $n=227$, Hündinnen: $n=281$ ) bzw. von der Futteraufnahme (nüchtern: $n=140$, nicht nüchtern: $n=368$ ); Unterschiede signifikant bei $\mathrm{p}<0,0001$

Table 6 Parameters with reference ranges (calculated as 95\%-percentile interval) depending on sex (male: $\mathrm{n}=227$, female: $\mathrm{n}=281$ ) or feed intake (fasting: $\mathrm{n}=140$, not fasting: $\mathrm{n}=368$ ), respectively; differences significant at $\mathrm{p}<0,0001$

\begin{tabular}{|c|c|c|c|}
\hline \multirow{2}{*}{\begin{tabular}{|l} 
Parameter \\
Geschlechtsabhängigkeit
\end{tabular}} & \multirow{2}{*}{$\begin{array}{l}\text { Maß- } \\
\text { einheit }\end{array}$} & \multicolumn{2}{|c|}{ Referenzbereich } \\
\hline & & Rüden & Hündinnen \\
\hline$a^{-}$-Amylase ${ }^{1}$ & $\mathrm{U} / \mathrm{I}$ & (+) 364-1434 & $349-1181$ \\
\hline Lipase $^{1}$ & $\mathrm{U} / \mathrm{I}$ & $(-)$ 63-597 & $83-668$ \\
\hline Leukozyten $^{2}$ & $G / I$ & (+) 4,9-16,1 & $4,1-15,7$ \\
\hline Lymphozyten $^{2}$ & $\mathrm{G} / \mathrm{I}$ & (-) $0,51-4,19$ & $0,56-4,12$ \\
\hline Fütterungsabhängigkeit & & nüchtern & gefüttert \\
\hline Serumgallensäuren $^{1}$ & $\mu \mathrm{mol} / \mathrm{l}$ & $(-)$ 0-17 & $0-65$ \\
\hline Triglyzeride $^{1}$ & $\mathrm{mmol} / \mathrm{l}$ & (+) 0,31-3,77 & $0,32-3,09$ \\
\hline Harnstoff $^{1}$ & $\mathrm{mmol} / \mathrm{l}$ & (-) 2,9-10,6 & $3,4-10,4$ \\
\hline
\end{tabular}




\section{Diskussion}

Das Ziel dieser Arbeit war Referenzwerte von Blutparametern und klinischchemischen Parametern von adulten Hunden zu erstellen. Die adulten Hunde waren zwischen 1 - 9 Jahre alt. Die Ergebnisse zeigten bei vielen in der Literatur bereits beschriebenen Referenzwerten eine Übereinstimmung. Dennoch wiesen einige Blutparameter Abweichungen zu den publizierten Referenzwerten auf. Der berechnete Referenzwert des Cell-Dyns der eosinophilen Granulozyten liegt in dieser Studie bei einem Maximum von 1,13 G/l und ist damit deutlich höher als in der bereits bestehenden Literatur. Die Parallelmessung durch mikroskopische Auswertung der Differenzialblutbilder ergab ebenfalls einen hohen Referenzwert der eosinophilen Granulozyten (0 1,28 G/l). Direkte Vergleiche mit Referenzwerten aus anderen Quellen sind sehr schwierig, da Messmethoden, Qualitätskontrollen und die Tierpopulationen oft nicht bekannt sind. In dieser Studie wurde vor der Blutabnahme bei jedem Tier eine klinische Allgemeinuntersuchung durchgeführt, hierbei konnte eine endogene Parasiteninfektion nicht ausgeschlossen werden. Entwurmung der Hunde fand wenigstens einmal im Jahr statt. Allergien konnten nur bei Tieren, deren Anamnese bekannt war, ausgeschlossen werden. Bei einigen Hunden aus den Tierheimen war der Allergiestatus sowie die letzte Entwurmung unbekannt. Die Privat- und die Laborhunde wurden durch den Besitzer oder durch einen Tierarzt mindestens einmal im Jahr entwurmt. Bei diesen Tieren waren keine Allergien bekannt. Da hypothetisch die eosinophilen Granulozyten bei Tierheimhunden durch die ständig wechselnden Populationen, unregelmäßige Entwurmung und oft schlechte Hygiene erhöht sein könnten, wurden auch Referenzwerte ohne diese Hunde berechnet. Das Resultat zeigt auch hier einen deutlich höheren oberen Referenzwert (1,12 G/l) als in der Literatur von KRAFT \& DÜRR (2005) beschrieben. Die Möglichkeit, dass dieser hohe Referenzwert durch eine Eosinophilie des Deutschen Schäferhundes entstand, kann ausgeschlossen werden, da in dieser Studie bei nur einem Schäferhund eine Konzentration von eosinophilen Granulozyten von 1,08 G/l gemessen wurde. Der Klimawandel und die dadurch günstigeren Lebensbedingungen der Parasiten sowie das 
Einschleppen von Parasiten, die sonst nur in südlicheren Regionen vorkamen, können möglicherweise die Ursache für den ermittelten Referenzwert sein. Ebenso kann die Zunahme von Allergien beim Hund auch ein mögliches Argument darstellen, denn auch beim Menschen wird eine Allergiezunahme dokumentiert (ASCHER, 2006, HERMANN-KUNZ, 2000, HEIL-FRANKE, 2002, Meyer, 2008). NEURER (2005) prüfte in seiner Studie die Zuverlässigkeit des Cell-Dyns 3500 und kam zu dem Ergebnis, das er eine hohe Präzision aufgrund niedriger Varianzkoeffizienten zeigte. Die eosinophilen Granulozyten wurden vom Cell-Dyn 3500 zuverlässig ermittelt. Diese Zuverlässigkeit konnten NEURER (2005) und LILLEHÖÖK (2011) bei den basophilen Granulozyten nicht feststellen, da diese nur in geringer Anzahl im Blut vorliegen. NEURER (2005) verzichtete auf eine statistische Auswertung.

Die Monozytenzahlen (1,29 G/1 Cell-Dyn, 0,92 G/1 mikroskopisches Differentialblutbild) sind ebenfalls höher als die Referenzwerte von KRAFT \& DÜRR (2005) (0,5 G/l) sowie bei WILLARD \& TVEDTEN (2004) (0,43 G/l). Bei GRETHER (2008) (0,2 - 0,9 G/l) war nur ein sehr geringer Unterschied zu verzeichnen. Eine Altersabhängigkeit wird von WESKAMP (1993) vermutet, sie beschreibt auch unterschiedliche Referenzwerte $\mathrm{zu}$ verschiedenen Fütterungszeiten. Es konnte in dieser Studie weder eine signifikante Abhängigkeit bezüglich des Alters, noch bezüglich des Fütterungszeitpunktes festgestellt werden.

Bei der Glutamatdehydrogenaseaktivität wurde eine signifikante Abhängigkeit vom Alter festgestellt. Bei jungen Hunden unter einem Jahr ist der Referenzwert niedriger als bei adulten Hunden. Die GLDH reagiert sehr empfindlich bei Lebererkrankungen, da liegt der Verdacht nahe, dass möglicherweise die Leber älterer Hunde stärker belastet ist und eventuell auch schon Leberschäden vorliegen können und dies ein Grund für die signifikante Abhängigkeit vom Alter sein kann. In dieser Studie liegt der Referenzwert bei 1 - $12 \mathrm{U} / \mathrm{l}$ und ist damit doppelt so hoch wie in der Literatur von KRAFT \& DÜRR (2005) angegeben. Bei ihnen liegt der obere Referenzbereich bei 6 U/1. Ein kurzzeitiger Anstieg dieses Parameters bis 15 G/l muss nicht pathologisch sein, zudem stellen KRAFT \& DÜRR (2005) die Aussagekraft der GLDH bei 
Haustieren in Frage. Um eine Verfälschung der Referenzwerte auszuschließen, da Anamnese und mögliche Vorerkrankungen der Tierheimhunde oft nicht bekannt waren, wurden zum Vergleich Referenzwerte ohne die Tierheimhunde erstellt. Es konnten jedoch keine signifikanten Unterschiede der beiden Referenzwerte festgestellt werden.

Die Serumaktivität der alkalischen Phosphatase (AP) ist ebenso wie die Serumkonzentration der Triglyceride ein altersabhängiger Blutparameter. Eine Signifikanz konnte in dieser Studie festgestellt werden und bestätigt damit auch die Ergebnisse von DERESER (1989) und KUHL et al. (2000), die ebenfalls eine Altersabhängigkeit der AP nachwiesen. Eine Signifikanz für den Faktor Alter und Geschlecht zeigte der Parameter Lipaseaktivität. Der Lipasewert älterer Hunde ist höher und bei Rüden signifikant niedriger. In der Literatur findet man sehr unterschiedliche Referenzwertangaben, wobei ein sehr hoher oberer Wert von DERESER (1989) mit 801 U/1 und ein niedriger oberer Wert mit 300 U/l von KRAFT \& DÜRR (2005) angegeben wird. In dieser Studie wurde ein oberer Referenzbereich von $563 \mathrm{U} / \mathrm{l}$ ermittelt und liegt damit genau zwischen den Ergebnissen von DERESER (1989) und KRAFT \& DÜRR (2005).

Der Referenzwert der Serumgallensäuren (SGS) $(0-18 \mu \mathrm{mol} / \mathrm{l})$ stimmte mit dem in der Literatur von KRAFT \& DÜRR (2005) bereits ermittelten Referenzwert fast überein. Sie ist stark abhängig von der letzten Nahrungsaufnahme und wurde für diesen Faktor signifikant getestet. Der postprandiale obere Referenzwert von KRAFT \& DÜRR (2005) liegt bei 40 $\mu \mathrm{mol} / \mathrm{l}$. Durch die stark variierenden Messwerte bei postprandialer Blutabnahme dürfen nur die SGS von nüchternen Hunden interpretiert werden. Es wird allgemein empfohlen, dass die letzte Nahrungsaufnahme vor der Blutabnahme mindestens 10 - 12 Stunden her sein sollte.

In dieser Studie sind die Referenzwerte von ALT (96 U/l), Bilirubin 5,5 $\mu \mathrm{mol} / \mathrm{l})$, CK (268 U/l) und Kreatinin $(119 \mu \mathrm{mol} / \mathrm{l})$ höher als bei KRAFT \& DÜRR (2005), wobei diese Werte bis auf die ALT niedriger sind als die in der Literatur beschriebenen Referenzbereiche von WILLARD \& TVEDTEN (2004). 
Die aktivierte partielle Thromboplastinzeit hat in dieser Studie einen Referenzwert von 9,6 - 14,9 Sekunden. Weder die Referenzbereiche von MISCHKE \& NOLTE (1999) noch die von REGEL (2002) und GREFFÉ (2010) konnten in dieser Studie bestätigt werden. MISCHKE \& NOLTES (1999) oberer Referenzbereich ist nur 1,4 Sekunden kürzer und REGEL (2002) liegt mit 2 Sekunden noch weiter von dem in dieser Studie ermittelten Referenzwert weg. Nur BAUER et al. (2009) hat einen Referenzwert von 10 14,3 Sekunden ermittelt, der sich mit dem aus dieser Studie vergleichen lässt.

Der Referenzbereich der Thrombinzeit liegt in dieser Studie liegt zwischen 8,23 - 16,76 Sekunden. Es ist bei einem Vergleich mit anderen Referenzwerten wichtig, dass das Testreagenz gleich ist. MISCHKE \& NOLTE (1999) geben in der Literatur Referenzbereiche an, die mit dem gleichen Testreagenz wie in dieser Studie ermittelt worden sind. Hier kann aber nur der obere Referenzbereich von MISCHKE \& NOLTE (1999) gerade noch bestätigt werden, da dieser bei 18 Sekunden liegt. Der Referenzbereich von REGEL (2002) liegt bei 9 - 12 Sekunden. Hier ist der untere Referenzbereich dem in dieser Studie ermittelten Referenzbereich sehr nahe und kann bestätigt werden. Es ist bei einem Vergleich $\mathrm{zu}$ berücksichtigen, dass auch die einzelnen Reagenzchargen, die angewandte Methode, die Ionenstärke, die Thrombinaktivität und die Plasmakonzentration $\mathrm{zu}$ unterschiedlichen Ergebnissen führen können (KRAFT \& DÜRR 2005). Die aus dieser Studie resultierenden Referenzbereiche dienen als Orientierungshilfe, wenn die durchgeführte Bestimmung der Thrombinzeit von der hier angewandten Methode und Reagenz abweicht.

Bei dem optimierten Quick-Test ist kein Vergleich der Referenzwerte mit REGEL (2002) möglich, da der damals verwendete Puffer nicht mehr erhältlich ist. In dieser Studie ist der Referenzbereich 9,5 - 22,0 Sekunden. 


\section{Zusammenfassung}

Blutuntersuchungen in der Tiermedizin sind wichtige zusätzliche Methoden, die zur Diagnosefindung oder Diagnosesicherung verwendet werden. Für eine möglichst genaue Interpretation eines Blutwertes ist es wichtig, einen definierten Referenzbereich zu haben. Durch die Vielzahl unterschiedlicher Messmethoden und Messgeräte müssen gegebenenfalls unterschiedliche Referenzbereiche bestimmt werden. In dieser Studie wurde eine große Anzahl von mehr als 500 klinisch gesunder Hunde unterschiedlicher Rassen und Alter zur Evaluierung neuer Referenzwerte untersucht. Neben den wichtigsten Lageund Streuparametern wurden zusätzlich Abhängigkeitsanalysen mit den Faktoren Alter, Fütterung und Geschlecht durchgeführt. Die Referenzwertbestimmung erfolgte durch das Analysieren der Blutwerte von adulten Hunden. Der wissenschaftliche Vorteil dieser Studie ist die große Anzahl der getesteten und ausgewerteten Tiere.

Das Resultat dieser Studie zeigt, dass die hämatologischen und klinischchemischen Referenzwerte zwar zum größten Teil aber nicht vollständig mit den in der Literatur veröffentlichten Referenzbereichen übereinstimmen. Deutliche Abweichungen fielen bei den Blutparametern eosinophile Granulozyten, basophile Granulozyten und Monozyten auf. Ein erschwerender Faktor ist, dass auch diese Referenzwerte nur mit anderen Referenzbereichen verglichen werden können, wenn die Geräte, sowie die Messmethoden in der Literatur angegeben werden. Abschließend lässt sich sagen, dass Referenzwerte immer wieder neu bestimmt werden müssen, sobald neue Geräte oder Messmethoden verwendet werden. 


\section{Summary}

Blood tests in veterinary medicine are important additional methods that can be used for diagnosis or confirm it. For the most accurate interpretation of a blood value, it is important to have a defined reference range. Due to the variety of different measurement methods and instruments different reference ranges are determined. In this study, a large number of more than 500 clinically healthy dogs of different breeds and ages to evaluate new reference values was investigated. Besides the most important position and scattering parameters dependence analyzes were performed with the factors of age, gender, and feeding. The reference values were determined by analyzing the blood parameters of the adult dogs. The scientific benefit of this study is the large number of animals tested and evaluated.

The result of this study shows that the haematological and clinical chemistry reference values for the most part but not completely agree with those published in the literature. Significant deviations from published reference ranges were found for the blood parameters eosinophils, basophils and monocytes. A complicating factor is that these reference values can only be compared to other reference values if the devices and the methods of measurement are given in the literature. It can be concluded that reference ranges must always be redefined as new devices or methods of measurement are used. 


\section{Literaturverzeichnis}

Abbott Diagnostika.Cell-Dyn 3500 System. Bedienungsanleitung SoftwareRevision J. 77-1566/R5 1. Auflage. Germany: Abbott Laboratories; 2000; 3-38,4$21,5-150,6-63,11-8$,

Abbrederis, N. Validierung von Laborparametern zur Diagnose einer DIC beim Hund [Dissertation]. München: Ludwig-Maximilians-Universität; 2005.

Ahne W, Liebich HG, Stroher M, Wolf E, König HE. Zoologie. 1. Auflage. Stuttgart: Schattauer Verlag; 2000; 294.

Asher I. Worldwide time in the prevalence of symptoms of asthma, allergic rhinoconjunctivitis, and eczema in childhood. ISAAC Phases One and Three repeat multicountry cross-sectional surveys. Lancet 2006; 368:733.

Backhaus K, Erichson B, Plinke W, Weiber R. Multivariate Analysemethoden. 8th ed. Berlin: Springer Verlag; 1996; XVII-XXIII, 56-7.

Bauer N, Eralp O, Moritz A. Reference intervals and method optimization for variables reflecting hypocoagulatory and hypercoagulatory states in dogs using the STA Compact automated analyzer. J Vet Diagn Invest 2009; 21(6), 803-14.

De Gruyter, W., Braun, N., Brehmer, A., Diehm, C., Dietz, K., Dörner, K., et al. Pschyrembel Klinisches Wörterbuch, 259. Auflage. Berlin: de Gryter Verlag; $2002 ; 1420$.

Dereser R. Blutchemische Referenzbereiche in der Labordiagnostik des Hundes [Dissertation]. München: Ludwig-Maximilians-Universität; 1989.

Eimer E. Varianzanalyse, 1st ed. Stuttgart, Berlin, Köln, Mainz: Kohlhammer Verlag; 1978; 7.

Engelhardt, W., Breves, G. Physiologie der Haustiere. 1. Auflage. Stuttgart: Enke 
Verlag; 2000; 118-20,148,192-3,221,264,292-3,462-4.

Fennell, J. Mit Hunden sprechen. 12.Auflage. Berlin: Ullstein Verlag; 2008; 21-5.

Grether, B., 2008: Veterinärmedizinisches Labor der Universität Zürich. Vet.uzh. [homepage of the Internet]. Available from: http://www.vet.uzh.ch/Studium/vetmed/studiengang/jk5/Jk5_rotation_blockb_200 7_8.pdf

Guggenmoos-Holzmann I, Wernecke D. Medizinische Statistik, 1st ed. Berlin: Blackwell Wissenschaftsverlag; 1995; 93-7.

Hartmann K. Referenzbereiche in der Labordiagnostik der Katze [Dissertation]. München: Ludwig-Maximilians-Universität; 1990.

Heil-Franke G, Hunsinger B. Spezifische Immuntherapie (SIT) beim Hund: Einfluss von Tierhalter, Art und Anzahl der Allergene. Kleintierpraxis. 2002; 47:483-7.

Henry RJ, Cannon DC, Winkelman JW. Normal values and the use of laboratory results for the detection of disease. Clinical Chemistry - Priniciples and Technics. 2nd ed. New York: Harper\&Row.1974; 343-71.

Hermann-Kunz E. Allergische Krankheiten in Deutschland Ergebnisse einer repräsentativen Studie. Bundesgesundheitsbl - Gesundheitsforsch Gesundheitsschutz. 2000; 43:400-6.

Hettich, A., Eberle H. Mikro 120 Hettich Laborzentrifuge. Bedienungsanleitung der Hettich GmbH. Tuttlingen; 2008; 6-51.

Holcak S., Riehs, K. Entwicklung einer Schnittstelle mittels LabVIEW für einen Hitachi 911 Automatic Analyser [Diplomarbeit]. Wien: DoA-Consulting; 2003.

Kraft W, Dürr UM. Klinische Labordiagnostik in der Tiermedizin. 6th ed. 
Stuttgart: Schattauer Verlag; 2005; 1-4,13-7,23-4,57,67-9,76-8,81-5,125-6,130$4,141,149,151-8,161,171,210,265-7,278,280-4,310,507-16$.

Kreutzig T. Kurzlehrbuch Biochemie. 10. Auflage. München, Jena: Urban \& Fischer Verlag; 2001; 33.

Kuhl, S., Mischke, R., Lund, C., Günzel-Apel, A.-R. Referenzwerte klinischchemischer Blutparameter bei Hundewelpen in den ersten acht Wochen. Dtsch Tierärztl Wschr 107. 2000; 11:438-443.

Laufner W. Beschreibende Statistik für Betriebswirte. 1st ed. Dortmund: Fachhochschule Dortmund; 2000; Modul 03:2, 79.

Lehninger AL, Nelson DL, Cox MM. Prinzipien der Biochemie. 2. Auflage. Heidelberg, Berlin, Oxford: Spektrum Akademischer Verlag; 1998; 102$5,221,230-5,485,600-3,703,768-74,853$.

Lilliehöök I, Tvedten HW. Errors in basophil enumeration with 3 veterinary hematology systems and observations on occurrence of basophils in dogs. ASVCP 2011; 40(4):450-8.

Meffert H. Marktforschung, 2nd ed. Wiesbaden: Gabler Verlag; 1992; 300.

Meyer R.. Zunahme von Allergien bei Kindern. Kinderumweltgesundheit [homepage of the Internet]. Köln: Deutsches Ärzteblatt 2008; Available from: http://www.kinderumweltgesundheit.de/KUG/index2/pdf/dokumente/50053_1.

Michl, M. Hämatologie. 1. Auflage. München: Elsevier GmbH; 2005; 10-5.

Mischke, R., Nolte. I. Hämostasediagnostik beim Hund: 2. Prinzip, Technik und Referenzbereich verschiedener Untersuchungsverfahren: Prakt Tierarzt.1999; 80(10):836-55. 
Mischke, R., Nolte. I. Optimization of prothrombin time measurements in canine plasma. Am. J. of Vet. 1997; 58(3): 236-241.

Neuerer, F. Evaluation des vollautomatischen Hämatologiegerätes Cell-Dyn 3500 im klinischen Einsatz bei Hund und Katze [Dissertation]. München: LudwigMaximilians-Universität; 2005.

Notfalllabor [homepage of the Internet]. Berlin: Instrumentation Laboratory GmbH: IL GEM® Premier 3000.c2002-09[updated 16.12.2008]. Available from: http://notfalllabor.de/Medizinische-Analysegeraete/1-310-10440-0-0-0-10-0.html.

Regel, A. Die disseminierte intravasale Gerinnung beim Hund [Dissertation]. München: Ludwig-Maximilians-Universität; 2002.

Schach S, Schäfer T. Regressions- und Varianzanalyse, 1st ed. Berlin, Heidelberg, New York: Springer Verlag; 1978; $172 \mathrm{ff.}$

Teske E, Rothuizen J, De Bruijne JJ, Rijnberk AA. Corticosteroid induced alkaline phosphatase isoenzyme in the diagnosis of canine hypercorticism. Vet Rec 1989; 125, 12-14.

Vogel Medizinische Technik und Elektronik [homepage of the Internet].Gießen: Blutausstrichfärbeautomat „Hema-Tek“; 2009: Available from: http://www.vogelgiessen.de/frameset.htm.

Weskamp M. Referenzbereiche in der Labordiagnostik des Hundes: Glucose, Harnstoff, Cholesterin, Triglyceride und Hämatologie [Dissertation].

München: Ludwig-Maximilians-Universität; 1993.

Wiesner E, Ribbeck R. Lexikon der Veterinärmedizin A-Z, 4th ed.

Stuttgart: Enke im Hippokrates Verlag; 2000; 41$4,118,154,193,233,260,263,427,445,574,577,587,619,830,893,964,1127,1218,123$ 8,1412 . 
Willard MD, Tvedten H. Small Animal Clinical Diagnosis by Laboratory Methods. 4th ed. Philadelphia: Imprint of Elsevier Science Limited; 2004; 35,125,153-7,262,297-8,533-7.

Zaman, Z., Blanckaert, N., Cobbaert, C., Gillery, P., Hagemann, P., Luthe H., et al. Multicentre evaluation of the Boehringer Mannheim/Hitachi 911 Analysis System. Journal of Automatic Chemistry. 1993;15 No 6:189-208. 


\section{Danksagung}

An dieser Stelle bedanke ich mich bei allen Personen, die zum Gelingen dieser Arbeit beigetragen haben.

Herrn Prof. Dr. med. vet. Johannes Hirschberger danke ich für die Überlassung des interessanten Themas und für die Unterstützung bei der Arbeit.

Frau Prof. Dr. med. vet. Katrin Hartmann sei an dieser Stelle mein Dank ausgesprochen für die Bereitstellung aller klinischen Einrichtungen und des Labors der Medizinischen Kleintierklinik der LMU München.

Den Mitarbeitern der Medizinischen Kleintierklinik, vor allem dem Laborteam, danke ich für die Hilfsbereitschaft und für die gute Zusammenarbeit.

Herzlich bedanke ich mich bei Frau Dr. med. vet. Carola Sauter-Louis für die statistische Beratung.

Ein Dankeschön geht auch an alle Hundehalter, die durch geeignete Tiere die Durchführung dieser Studie unterstützt haben.

Besonders lobenswert sind folgende Personen:

Sylvia Meierhans bin ich zum Dank verpflichtet, da sie mir jederzeit mit Rat und Tat auch über große Entfernungen und lange Jahre beistand. Ebenso danke ich David Bennett und Marco Steinem für den englisch- und deutschsprachlichen Feinschliff.

Ein Dankeschön an Nadine Bohn für die Formatierung und Anne Hunold für die Anleitungsrecherche.

Einen Dank an meinen Freundeskreis, der mir treu geblieben ist, sowie auch meinen Tieren die immer für einen passenden Ausgleich gesorgt haben.

Ein besonderer und großer Dank gilt meinen Eltern für die Motivation und Unterstützung, ohne die diese Arbeit wahrscheinlich nicht möglich gewesen wäre. 